\title{
Synthesis of Carbamoyl Azides from Primary Amines and Carbon Dioxide under Mild Conditions
}

\section{Eduardo García-Egido, Miryam Fernández-Suárez and Luis Muñoz*}

Departamento de Química Orgánica, Universidade de Vigo. E-36310 Vigo, Spain.

lmunoz@uvigo.es

Experimental procedures and characterization data

${ }^{1} \mathrm{H}$ and ${ }^{13} \mathrm{C}$ NMR $\left(\mathrm{CDCl}_{3}, 400.13 / 100.62 \mathrm{MHz}\right)$ spectra of 2a

${ }^{1} \mathrm{H}$ and ${ }^{13} \mathrm{C}$ NMR $\left(\mathrm{CDCl}_{3}, 400.13 / 100.63 \mathrm{MHz}\right)$ spectra of $\mathbf{2 b}$

${ }^{1} \mathrm{H}$ and ${ }^{13} \mathrm{C}$ NMR $\left(\mathrm{CDCl}_{3}, 400.13 / 100.61 \mathrm{MHz}\right)$ spectra of 2c

${ }^{1} \mathrm{H}$ and ${ }^{13} \mathrm{C}$ NMR $\left(\mathrm{CDCl}_{3}, 400.13 / 100.63 \mathrm{MHz}\right)$ spectra of 2d

${ }^{1} \mathrm{H}$ and ${ }^{13} \mathrm{C}$ NMR $\left(\mathrm{CDCl}_{3}, 400.13 / 100.63 \mathrm{MHz}\right)$ spectra of $\mathbf{2 e}$

${ }^{1} \mathrm{H}$ and ${ }^{13} \mathrm{C}$ NMR $\left(\mathrm{CDCl}_{3}, 400.13 / 100.61 \mathrm{MHz}\right)$ spectra of $\mathbf{2 f}$

${ }^{1} \mathrm{H}$ and ${ }^{13} \mathrm{C}$ NMR $\left(\mathrm{CDCl}_{3}, 400.13 / 100.62 \mathrm{MHz}\right)$ spectra of $\mathbf{2 g}$

${ }^{1} \mathrm{H}$ and ${ }^{13} \mathrm{C}$ NMR $\left(\mathrm{CDCl}_{3}, 400.13 / 100.62 \mathrm{MHz}\right)$ spectra of $\mathbf{2 h}$

${ }^{1} \mathrm{H}$ and ${ }^{13} \mathrm{C}$ NMR $\left(\mathrm{CDCl}_{3}, 400.13 / 100.62 \mathrm{MHz}\right)$ spectra of $\mathbf{2 i}$

${ }^{1} \mathrm{H}$ and ${ }^{13} \mathrm{C}$ NMR $\left(\mathrm{CDCl}_{3}, 400.16 / 100.63 \mathrm{MHz}\right)$ spectra of $\mathbf{2 j}$

${ }^{1} \mathrm{H}$ and ${ }^{13} \mathrm{C}$ NMR $\left(\mathrm{CDCl}_{3}, 400.13 / 100.62 \mathrm{MHz}\right)$ spectra of $\mathbf{2 k}$

${ }^{1} \mathrm{H}$ and ${ }^{13} \mathrm{C}$ NMR $\left(\mathrm{CDCl}_{3}, 400.13 / 100.62 \mathrm{MHz}\right)$ spectra of $2 \mathbf{l}$

${ }^{1} \mathrm{H}$ and ${ }^{13} \mathrm{C}$ NMR $\left(\mathrm{CDCl}_{3}, 400.13 / 100.61 \mathrm{MHz}\right)$ spectra of $\mathbf{2 m}$

${ }^{1} \mathrm{H}$ and ${ }^{13} \mathrm{C}$ NMR $\left(\mathrm{CDCl}_{3}, 400.13 / 100.62 \mathrm{MHz}\right)$ spectra of $\mathbf{2 n}$

${ }^{1} \mathrm{H}$ and ${ }^{13} \mathrm{C}$ NMR $\left(\mathrm{CDCl}_{3}, 400.13 / 100.62 \mathrm{MHz}\right)$ spectra of $2 \mathbf{o}$

${ }^{1} \mathrm{H}\left(\mathrm{CDCl}_{3}, 400.13 \mathrm{MHz}\right)$ and ${ }^{13} \mathrm{C}$ NMR $\left(\mathrm{CD}_{3} \mathrm{OD}, 100.62 \mathrm{MHz}\right)$ spectra of 4

${ }^{1} \mathrm{H},{ }^{31} \mathrm{P}$ and ${ }^{13} \mathrm{C}$ NMR $\left(\mathrm{CDCl}_{3}, 400.13 / 161.97 / 100.62 \mathrm{MHz}\right)$ spectra of 5

${ }^{1} \mathrm{H}$ and ${ }^{13} \mathrm{C}$ NMR $\left(\mathrm{CDCl}_{3}, 400.13 / 100.62 \mathrm{MHz}\right)$ spectra of 6

Ortep plot of $\mathbf{2 e}$

Ortep plot of 4
S2-S8

S9

S10

S11

S12

S13

S14

S15

S16

S17

S18

S19

S20

S21

S22

S23

S24

S25

S26

S27

S28 


\section{General procedure for the synthesis of carbamoyl azides from primary amines}

A mixture of $\mathrm{NaN}_{3}(0.200 \mathrm{~g}, 3.08 \mathrm{mmol}, 302 \mathrm{~mol} \%)$ and DPPA (0.280 g, $0.22 \mathrm{~mL}$, $1.00 \mathrm{mmol})$ in dry acetonitrile $(10 \mathrm{~mL})$ was cooled in a dry ice/acetone bath at -41 ${ }^{\circ} \mathrm{C}$. Carbon dioxide was slowly bubbled through the mixture until saturation was achieved. A solution of the amine (1.06 mmol, $106 \mathrm{~mol} \%)$ and PhTMG (0.220 g, $1.15 \mathrm{mmol}, 112 \mathrm{~mol} \%)$ in dry acetonitrile $(15 \mathrm{~mL})$ was added dropwise (1.5-2.0 h). Once the addition was finished, the stream of $\mathrm{CO}_{2}$ was stopped and the mixture was stirred under a carbon dioxide atmosphere, allowing the temperature to rise to room temperature overnight $(14-17 \mathrm{~h})$. The mixture was dissolved in EtOAc $(150 \mathrm{~mL})$ and the solution was washed with water $(3 \times 15 \mathrm{~mL})$ and with $5 \%$ aq $\mathrm{HCl}(3 \times 15 \mathrm{~mL})$. The resulting organic phase was dried with anhydrous $\mathrm{Na}_{2} \mathrm{SO}_{4}$, filtered and concentrated under reduced pressure. The residue was purified by flash chromatography.

Pentane-1,5-diyldicarbamoyl azide (2a). Following the general procedure, 2.04 mmol of DPPA and $2.30 \mathrm{mmol}$ of PhTMG were used. Thus, starting from $1.00 \mathrm{mmol}$ of pentane-1,5-diamine, after purification by flash chromatography (EtOAc/hexanes from 1:9 to 1:1), the product was obtained as a white solid $(0.160 \mathrm{~g}, 69 \%$ yield). $R f$ : 0.60 (hex/EtOAc 1:1). Mp: $115.8{ }^{\circ} \mathrm{C}(\mathrm{dec}) .{ }^{1} \mathrm{H}$ NMR $\left(\mathrm{CDCl}_{3}, 400.13 \mathrm{MHz}\right.$ ) (two rotamers, ratio 93:7) $\delta 5.12$ (br s, $2 \mathrm{H}$ ), 3.25 (q, 3.7H, $J=6.5 \mathrm{~Hz}$ ), 3.13 (q, $0.3 \mathrm{H}, J=$ $6.5 \mathrm{~Hz}), 1.55$ (q, 4H, $J=7.0 \mathrm{~Hz}), 1.36(\mathrm{~m}, 2 \mathrm{H}) ;{ }^{13} \mathrm{C} \mathrm{NMR}\left(\mathrm{CDCl}_{3}, 100.62 \mathrm{MHz}\right)$ (major rotamer) $\delta 156.3,40.4$, 28.7, 23.1; FTIR (KBr) $v_{\max } 3256$ (br), 2178, 2142, 1686, 1668, 1539, 1258, 1225 (br) $\mathrm{cm}^{-1}$; HRMS (FAB+, mNBA matrix): calcd for $\mathrm{C}_{7} \mathrm{H}_{13} \mathrm{~N}_{8} \mathrm{O}_{2}$ 241.1161, found 241.1154.

Benzylcarbamoyl azide (2b). Following the general procedure, and starting from $1.00 \mathrm{mmol}$ of benzylamine, after purification by flash chromatography (EtOAc/hexanes 1:9), the product was obtained as a white solid ( $0.129 \mathrm{~g}, 74 \%$ yield). Rf: 0.55 (hex/EtOAc 3:1). Mp: 79.6-80.8 ${ }^{\circ} \mathrm{C} .{ }^{1} \mathrm{H}$ NMR $\left(\mathrm{CDCl}_{3}, 400.13 \mathrm{MHz}\right.$ ) (two rotamers, ratio 92:8) $\delta 7.38-7.26(\mathrm{~m}, 5 \mathrm{H}), 5.39(\mathrm{br} \mathrm{s}, 0.9 \mathrm{H}), 5.23(\mathrm{br} \mathrm{s}, 0.1 \mathrm{H}), 4.43(\mathrm{~d}$, $1.8 \mathrm{H}, J=5.9 \mathrm{~Hz}), 4.34(\mathrm{~d}, 0.2 \mathrm{H}, J=5.9 \mathrm{~Hz}) ;{ }^{13} \mathrm{C} \mathrm{NMR}\left(\mathrm{CDCl}_{3}, 100.63 \mathrm{MHz}\right)$ (major rotamer) $\delta$ 156.5, 137.2, 128.8, 127.9, 127.7, 45.1; FTIR (KBr) $v_{\max } 3339$ (br), 2163 (br), 1679 (br), 1529 (br), 1266, 1235, 735, 698, $613 \mathrm{~cm}^{-1}$; LRMS (EI) $\mathrm{m} / \mathrm{z}$ (relative intensity) 176 ( $\left.\mathrm{M}^{+}, 0.2\right), 147$ (78), 104 (67), 91 (100), 77 (21), 65 (21); HRMS (EI) calcd for $\mathrm{C}_{8} \mathrm{H}_{8} \mathrm{~N}_{4} \mathrm{O}\left(\mathrm{M}^{+}\right)$176.0698, found 176.0699.

2-(1-Cyclohexenyl)ethylcarbamoyl azide (2c). Following the general procedure, and starting from $1.00 \mathrm{mmol}$ of 2-(1-cyclohexenyl)ethylamine, after purification by 
flash chromatography (EtOAc/hexanes 1:9), the product was obtained as a white solid (0.161 g, 84\% yield). Rf: 0.65 (hex/EtOAc 3:1). Mp: 65.3-66.4 ${ }^{\circ} \mathrm{C} .{ }^{1} \mathrm{H}$ NMR $\left(\mathrm{CDCl}_{3}, 400.13 \mathrm{MHz}\right.$ ) (two rotamers, ratio 9:1) $\delta 5.47$ (s, 1H), 5.05 (br s, 1H), 3.31 $(\mathrm{q}, 1.8 \mathrm{H}, J=6.5 \mathrm{~Hz}), 3.19(\mathrm{q}, 0.2 \mathrm{H}, J=6.5 \mathrm{~Hz}), 2.14(\mathrm{t}, 2 \mathrm{H}, J=6.5 \mathrm{~Hz}), 1.99$ (d, 2H, $J=1.3 \mathrm{~Hz}), 1.91$ (s, 2H), 1.65-1.52 (m, 4H); ${ }^{13} \mathrm{C} \mathrm{NMR}\left(\mathrm{CDCl}_{3}, 100.61 \mathrm{MHz}\right)$ (major rotamer) $\delta 156.2$, 133.9, 124.1, 38.6, 37.5, 27.7, 25.2, 22.7, 22.2; FTIR (KBr) $v_{\max }$ 3290 (br), 2934 (br), 2174 (br), 2140 (br), 1712, 1678 (br), 1549 (br), 1249 (br) cm ${ }^{-1}$; LRMS (EI) m/z (relative intensity) $194\left(\mathrm{M}^{+}, 8\right), 162$ (30), 149 (28), 108 (100), 99 (37), 93 (46), 79 (59), 67 (42); HRMS (EI) calcd for $\mathrm{C}_{9} \mathrm{H}_{14} \mathrm{~N}_{4} \mathrm{O}\left(\mathrm{M}^{+}\right)$194.1168, found 194.1159.

Cyclohexylcarbamoyl azide (2d). Following the general procedure, and starting from $1.00 \mathrm{mmol}$ of cyclohexylamine, after purification by flash chromatography (EtOAc/hexanes from 1:19 to 1:9), the product was obtained as a white solid (0.126 g, 76\% yield). Rf: 0.45 (hex/EtOAc 9:1). Mp: 98.6-99.6 ${ }^{\circ} \mathrm{C} .{ }^{1} \mathrm{H} \mathrm{NMR}\left(\mathrm{CDCl}_{3}, 400.13\right.$ $\mathrm{MHz}$ (two rotamers, ratio 9:1) $\delta 4.98$ (br s, 0.9H), 4.85 (br s, $0.1 \mathrm{H}$ ), 3.60 (m, 0.9H), $3.31(\mathrm{~m}, 0.1 \mathrm{H}), 1.94(\mathrm{~m}, 2 \mathrm{H}), 1.74-1.59(\mathrm{~m}, 3 \mathrm{H}), 1.41-1.10(\mathrm{~m}, 5 \mathrm{H}) ;{ }^{13} \mathrm{C}$ NMR $\left(\mathrm{CDCl}_{3}, 100.63 \mathrm{MHz}\right.$ ) (major rotamer) $\delta 155.5,50.0$, 32.9, 25.3, 24.6; FTIR (KBr) $v_{\max } 3280$ (br), 2939, 2139 (br), 1711, 1677, 1542, 1278, $1231 \mathrm{~cm}^{-1}$; LRMS (EI) $\mathrm{m} / \mathrm{z}$ (relative intensity) $168\left(\mathrm{M}^{+}, 38\right), 162$ (100), 151 (16), 132 (20), 125 (83), 111 (15), 97 (24), 94 (22), 91 (42), 83 (55), 71 (56); HRMS (EI) calcd for $\mathrm{C}_{7} \mathrm{H}_{12} \mathrm{~N}_{4} \mathrm{O}\left(\mathrm{M}^{+}\right.$) 168.1011, found 168.1007 .

Homoveratrylcarbamoyl azide (2e). Following the general procedure, and starting from $1.00 \mathrm{mmol}$ of homoveratrylamine, after purification by flash chromatography (EtOAc/hexanes from 1:9 to 1:2), the product was obtained as a white solid (0.190 g, 77\% yield). $R f: 0.25$ (hex/EtOAc 2:1). Mp: 92.7-93.2 ${ }^{\circ} \mathrm{C} .{ }^{1} \mathrm{H}$ NMR $\left(\mathrm{CDCl}_{3}, 400.13\right.$ MHz) (two rotamers, ratio 19:1) $\delta 6.82(\mathrm{~d}, 1 \mathrm{H}, J=8.1 \mathrm{~Hz}$ ), 6.74-6.68 (m, 2H), 5.09 (br s, 0.9H), 4.91 (br s, 0.1H), $3.88(\mathrm{~s}, 3 \mathrm{H}), 3.87$ (s, 3H), 3.48 (q, $2 \mathrm{H}, J=6.8 \mathrm{~Hz}$ ), 3.37 (q, 2H, $J=6.8 \mathrm{~Hz}), 2.78(\mathrm{t}, 2 \mathrm{H}, J=6.9 \mathrm{~Hz}) ;{ }^{13} \mathrm{C} \mathrm{NMR}\left(\mathrm{CDCl}_{3}, 100.63 \mathrm{MHz}\right)$ (major rotamer) $\delta 156.4,149.0,147.7,130.5,120.6,111.7,111.2,55.9,55.8,42.2$, 35.2; FTIR (KBr) $v_{\max } 3284$ (br), 2136 (br), 1702, 1680, 1551, 1517, 1255, 1237, 1144, $1024 \mathrm{~cm}^{-1}$; LRMS (EI) $\mathrm{m} / \mathrm{z}$ (relative intensity) $250\left(\mathrm{M}^{+}, 41\right), 207$ (6), 164 (33), 151 (100), 137 (10), 107 (6); HRMS (EI) calcd for $\mathrm{C}_{11} \mathrm{H}_{14} \mathrm{~N}_{4} \mathrm{O}_{3}\left(\mathrm{M}^{+}\right)$250.1066, found 250.1073 .

Allylcarbamoyl azide (2f). Following the general procedure, and starting from 1.15 mmol of allylamine, after purification by flash chromatography (EtOAc/hexanes 1:9), the product was obtained as a volatile colorless oil (0.089 g, $71 \%$ yield). $R f: 0.65$ 
(hex/EtOAc 3:1). ${ }^{1} \mathrm{H}$ NMR $\left(\mathrm{CDCl}_{3}, 400.13 \mathrm{MHz}\right.$ ) (two rotamers, ratio 93:7) $\delta 5.81$ (m, $1 \mathrm{H}, J=17.1,10.9$ and $7.2 \mathrm{~Hz}$ ), 5.49 (br s, $1 \mathrm{H}$ ), 5.19 (ddd, $1 \mathrm{H}, J=17.1,2.8$ and $1.5 \mathrm{~Hz}$ ), 5.15 (ddd, $1 \mathrm{H}, J=10.9,2.6$ and $1.3 \mathrm{~Hz}$ ), 3.84 (tt, $1.9 \mathrm{H}, J=5.8$ and $1.5 \mathrm{~Hz}$ ), $3.74(\mathrm{~m}, 0.1 \mathrm{H}, J=4.5 \mathrm{~Hz}) ;{ }^{13} \mathrm{C} \mathrm{NMR}\left(\mathrm{CDCl}_{3}, 100.61 \mathrm{MHz}\right)$ (major rotamer) $\delta 156.4$, 133.2, 117.0, 43.4; FTIR (neat) $v_{\max } 3317$ (br), 2155 (br), 1695 (br), 1538 (br), 1229 (br) $\mathrm{cm}^{-1}$.

Isobutylcarbamoyl azide (2g). Following the general procedure, and starting from $1.00 \mathrm{mmol}$ of isobutylamine, after purification by flash chromatography (EtOAc/hexanes from 1:19 to 1:4), the product was obtained as volatile colorless oil (0.112 g, 80\% yield). $R f: 0.55$ (hex/EtOAc 4:1). ${ }^{1} \mathrm{H} \mathrm{NMR}\left(\mathrm{CDCl}_{3}, 400.13 \mathrm{MHz}\right.$ ) (two rotamers, ratio 9:1) $\delta 5.26$ (br s, $1 \mathrm{H}$ ), 3.06 (t, $1.8 \mathrm{H}, J=6.5 \mathrm{~Hz}$ ), 2.94 (t, $0.2 \mathrm{H}, J=6.5$ $\mathrm{Hz}), 1.78$ (m, 1H), 0.91 (d, 6H, $J=6.7 \mathrm{~Hz}) ;{ }^{13} \mathrm{C} \mathrm{NMR}\left(\mathrm{CDCl}_{3}, 100.62 \mathrm{MHz}\right.$ ) (major rotamer) $\delta$ 156.5, 48.4, 28.5, 19.8; FTIR (neat) $v_{\max } 3317$ (br), 2144, 1701 (br), 1229 $\mathrm{cm}^{-1}$.

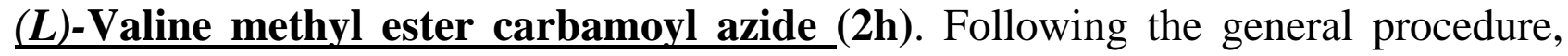
and starting from $1.00 \mathrm{mmol}$ of $(L)$-valine methyl ester hydrochloride and $2.30 \mathrm{mmol}$ of PhTMG, after purification by flash chromatography (EtOAc/hexanes from 1:7 to 1:4), the product was obtained as a yellowish oil ( $0.178 \mathrm{~g}, 90 \%$ yield). $R f: 0.43$ (hex/EtOAc 4:1). $[\alpha]^{\mathrm{D}}{ }_{20}+0.62$ (c 2.57, $\left.\mathrm{CHCl}_{3}\right) .{ }^{1} \mathrm{H} \mathrm{NMR}\left(\mathrm{CDCl}_{3}, 400.13 \mathrm{MHz}\right.$ ) (two rotamers, ratio 93:7) $\delta 5.72$ (br d, $1 \mathrm{H}, J=5.6 \mathrm{~Hz}$ ), 4.37 (dd, $0.9 \mathrm{H}, J=9.0$ and 4.8 $\mathrm{Hz}$ ), 4.04 (dd, 0.1H, $J=9.0$ and $4.8 \mathrm{~Hz}$ ), $3.72(\mathrm{~s}, 3 \mathrm{H}), 2.15(\mathrm{~m}, 1 \mathrm{H}), 0.94$ (d, 3H, $J=$ $6.8 \mathrm{~Hz}), 0.87(\mathrm{~d}, 3 \mathrm{H}, J=6.9 \mathrm{~Hz}) ;{ }^{13} \mathrm{C} \mathrm{NMR}\left(\mathrm{CDCl}_{3}, 100.62 \mathrm{MHz}\right.$ ) (major rotamer) $\delta$ 171.8, 156.5, 58.8, 52.3, 31.3, 18.0, 17. 5; FTIR (neat) $v_{\max } 3331$ (br), 2153 (br), 1754 (br), 1684 (br), 1506 (br), 1468, 1438, 1218, $1150 \mathrm{~cm}^{-1}$; HRMS (FAB+, mNBA matrix): calcd for $\mathrm{C}_{7} \mathrm{H}_{13} \mathrm{~N}_{4} \mathrm{O}_{3}$ 201.0988, found 201.0992.

(D)-Valine methyl ester carbamoyl azide (2i). Following the general procedure, and starting from $1.00 \mathrm{mmol}$ of $(L)$-valine methyl ester hydrochloride and $2.30 \mathrm{mmol}$ of PhTMG, after purification by flash chromatography (EtOAc/hexanes from 1:7 to 1:4), the product was obtained as a yellowish oil ( $0.178 \mathrm{~g}, 90 \%$ yield). $R f: 0.43$ (hex/EtOAc 4:1). [ $\alpha]_{20}^{\mathrm{D}}-0.45$ (c 2.23, $\mathrm{CHCl}_{3}$ ). Spectroscopic data identical to those of $\mathbf{2 h}$.

(L)-Glutamic acid dimethyl diester carbamoyl azide (2j). Following the general procedure, and starting from $1.00 \mathrm{mmol}$ of $(L)$-glutamic acid dimethyl diester hydrochloride and $2.30 \mathrm{mmol}$ of PhTMG, after purification by flash chromatography 
(EtOAc/hexanes from 1:4 to 1:2), the product was obtained as a yellowish oil (0.213 g, 88\% yield). Rf: 0.50 (hex/EtOAc 2:1). [ $\alpha]^{\mathrm{D}}{ }_{20}+22.16$ (c 2.57, $\mathrm{CHCl}_{3}$ ). ${ }^{1} \mathrm{H}$ NMR $\left(\mathrm{CDCl}_{3}, 400.16 \mathrm{MHz}\right.$ ) (two rotamers, ratio 93:7) $\delta 6.05$ (br d, 0.9H), 5.84 (br s, $0.1 \mathrm{H}), 4.46(\mathrm{dt}, 0.9 \mathrm{H}, J=8.1$ and $5.1 \mathrm{~Hz}), 4.25-4.22(\mathrm{~m}, 0.1 \mathrm{H}), 3.75(\mathrm{~s}, 3 \mathrm{H}), 3.67$ (s, 3H), 2.47-2.33 (m, 2H), 2.21 (m, 1H), 1.99 (m, 1H); $\left.{ }^{13} \mathrm{C} \mathrm{NMR} \mathrm{(CDCl} 3,100.63 \mathrm{MHz}\right)$ (major rotamer) $\delta 173.0,171.5,156.4,53.1,52.7,51.8,29.8,27.1$; FTIR (neat) $v_{\max }$ 3333 (br), 2146 (br), 1740 (br), 1714 (br), 1533 (br), 1439 (br), 1352, 1214 (br) cm ${ }^{-1}$; HRMS (FAB+, mNBA matrix): calcd for $\mathrm{C}_{8} \mathrm{H}_{13} \mathrm{~N}_{4} \mathrm{O}_{5}$ 245.0886, found 245.0889.

(L)-Tryptophan methyl ester carbamoyl azide (2k). Following the general procedure, and starting from $1.00 \mathrm{mmol}$ of $(L)$-tryptophan methyl ester hydrochloride and $2.30 \mathrm{mmol}$ of PhTMG, after purification by flash chromatography (EtOAc/hexanes from 1:4 to 1:2), the product was obtained as a yellowish foam (0.243 g, 86\% yield). Rf: 0.45 (hex/EtOAc 2:1). [ $\alpha]^{\mathrm{D}}{ }_{20}+111.27$ (c 1.54, $\mathrm{CHCl}_{3}$ ). ${ }^{1} \mathrm{H}$ NMR $\left(\mathrm{CDCl}_{3}, 400.13 \mathrm{MHz}\right.$ ) (two rotamers, ratio 93:7) $\delta 8.40(\mathrm{br} \mathrm{s}, 1 \mathrm{H}), 7.58(\mathrm{~d}, 1 \mathrm{H}$, $J=7.8 \mathrm{~Hz}$ ), 7.37 (d, 1H, $J=8.1 \mathrm{~Hz}$ ), 7.25-7.14 (m, 2H), 6.98 (s, 1H), 5.84 (br d, $0.9 \mathrm{H}, J=7.6 \mathrm{~Hz}), 5.67(\mathrm{~s}, 0.1 \mathrm{H}), 4.84(\mathrm{dt}, 0.9 \mathrm{H}, J=8.5$ and $5.5 \mathrm{~Hz}), 4.52(\mathrm{~m}, 0.1 \mathrm{H})$, 3.73 (s, 3H), 3.38-3.36 (m, 2H); ${ }^{13} \mathrm{C} \mathrm{NMR}\left(\mathrm{CDCl}_{3}, 100.62 \mathrm{MHz}\right) \delta 171.7,156.2$, 136.0, 127.2, 122.9, 122.2, 119.6, 118.2, 118.1, 111.3, 109.1, 54.3, 52.6, 27.5; FTIR (neat) $v_{\max } 3405$ (br), 2143 (br), 1741 (br), 1696 (br), 1518 (br), 1228 (br) cm ${ }^{-1}$; LRMS (EI) $\mathrm{m} / \mathrm{z}$ (relative intensity) $287\left(\mathrm{M}^{+}\right), 244$ (8), 130 (100); HRMS (EI) calcd for $\mathrm{C}_{13} \mathrm{H}_{13} \mathrm{~N}_{5} \mathrm{O}_{3}\left(\mathrm{M}^{+}\right) 287.1018$, found 287.1018.

(L)-Aspartic acid dibenzyl diester carbamoyl azide (2l). Following the general procedure, and starting from $1.00 \mathrm{mmol}$ of $(L)$-aspartic acid dibenzyl diester $p$ toluenesulfonate and $2.30 \mathrm{mmol}$ of PhTMG, after purification by flash chromatography (EtOAc/hexanes from 1:9 to 1:4), the product was obtained as a white solid (0.287 g, $76 \%$ yield). $R f: 0.70$ (hex/EtOAc 2:1). Mp: $73.2-74.4{ }^{\circ} \mathrm{C}$. [ $\alpha$ ]$^{\mathrm{D}}{ }_{20}+26.54$ (c 1.93, $\left.\mathrm{CHCl}_{3}\right) .{ }^{1} \mathrm{H}$ NMR $\left(\mathrm{CDCl}_{3}, 400.13 \mathrm{MHz}\right)$ (two rotamers, ratio 9:1) $\delta 7.40-7.28$ (m, 10H), 6.10 (br d, $1 \mathrm{H}, J=7.0 \mathrm{~Hz}$ ), $5.16(\mathrm{~s}, 2 \mathrm{H}), 5.08$ (s, 2H) 4.74 (dt, $0.9 \mathrm{H}, J=8.5$ and $4.5 \mathrm{~Hz}$ ), 4.50-4.46 (m, 0.1H), 3.10 (dd, $1 \mathrm{H}, J=17.3$ and $4.5 \mathrm{~Hz}$ ), 2.92 (dd, $1 \mathrm{H}, J=17.3$ and $4.5 \mathrm{~Hz}) ;{ }^{13} \mathrm{C}$ NMR $\left(\mathrm{CDCl}_{3}, 100.62 \mathrm{MHz}\right.$ ) (major rotamer) $\delta$ 170.3, 169.7, 156.5, 135.1, 134.9, 128.61, 128.56, 128.5, 128.4, 128.3, 128.2, 67.8, 67.0, 50.2, 36.3; FTIR (KBr) $v_{\max } 3306$ (br), 2184 (br), 2136 (br), 1734 (br), 1671 (br), 1533 (br), 1346, 1295, 1225 (br), 1157, $1130 \mathrm{~cm}^{-1}$; LRMS (EI) $\mathrm{m} / \mathrm{z}$ (relative intensity) $383\left(\mathrm{M}^{+}\right.$), 326 (6), 247 (13), 185 (14), 180 (13), 107 (77), 91 (100), 77 (7); HRMS (EI) calcd for $\mathrm{C}_{19} \mathrm{H}_{18} \mathrm{~N}_{4} \mathrm{O}_{5}\left(\mathrm{M}^{+}\right)$382.1277, found 382.1278. 
(L)-Isoleucine methyl ester carbamoyl azide (2m). Following the general procedure, and starting from $1.00 \mathrm{mmol}$ of $(L)$-isoleucine methyl ester hydrochloride and $2.30 \mathrm{mmol}$ of PhTMG, after purification by flash chromatography (EtOAc/hexanes from 1:9 to 1:4), the product was obtained as a yellowish oil (0.191 g, 90\% yield). Rf: 0.55 (hex/EtOAc 4:1). [ $\alpha]^{\mathrm{D}}{ }_{20}+20.79$ (c 1.82, $\mathrm{CHCl}_{3}$ ). ${ }^{1} \mathrm{H}$ NMR $\left(\mathrm{CDCl}_{3}, 400.13 \mathrm{MHz}\right.$ ) (two rotamers, ratio 9:1) $\delta 5.87$ (br s, 0.9H), 5.73 (br s, 0.1H) 4.40 (dd, $1 \mathrm{H}, J=8.9$ and $4.9 \mathrm{~Hz}$ ), $3.72(\mathrm{~s}, 3 \mathrm{H}), 1.88(\mathrm{~m}, 1 \mathrm{H}, J=5.0 \mathrm{~Hz}), 1.39$ (m, $1 \mathrm{H}, J=4.8 \mathrm{~Hz}), 1.16(\mathrm{~m}, 1 \mathrm{H}, J=7.2 \mathrm{~Hz}), 0.91-0.87(\mathrm{~m}, 6 \mathrm{H}) ;{ }^{13} \mathrm{C} \mathrm{NMR}\left(\mathrm{CDCl}_{3}\right.$, $100.61 \mathrm{MHz}$ ) (major rotamer) $\delta 171.7,156.3,58.1,52.2,37.9,24.9,15.3,11.4$; FTIR (neat) $v_{\max } 3334$ (br), 2967, 2149 (br), 1749 (br), 1701 (br), 1534 (br), 1229 (br), $1145 \mathrm{~cm}^{-1}$; LRMS (EI) $\mathrm{m} / \mathrm{z}$ (relative intensity) $214\left(\mathrm{M}^{+}, 5\right), 155$ (100), 88 (12), 71 (11); HRMS (EI) calcd for $\mathrm{C}_{8} \mathrm{H}_{14} \mathrm{~N}_{4} \mathrm{O}_{3}\left(\mathrm{M}^{+}\right)$214.1066, found 214.1075. HRMS (FAB+, mNBA matrix): calcd for $\mathrm{C}_{8} \mathrm{H}_{15} \mathrm{~N}_{4} \mathrm{O}_{5}$ 215.1144, found 215.1139.

(L)-Methionine methyl ester carbamoyl azide (2n). Following the general procedure, and starting from $1.00 \mathrm{mmol}$ of $(L)$-methionine methyl ester trifluoroacetate and $2.30 \mathrm{mmol}$ of PhTMG, after purification by flash chromatography (EtOAc/hexanes from 1:9 to 1:4), the product was obtained as a colorless oil (0.185 g, 81\% yield). Rf: 0.35 (hex/EtOAc 4:1). [ $\alpha]^{\mathrm{D}}{ }_{20}+56.50$ (c 1.88, $\left.\mathrm{CHCl}_{3}\right) .{ }^{1} \mathrm{H}$ NMR $\left(\mathrm{CDCl}_{3}, 400.13 \mathrm{MHz}\right.$ ) (two rotamers, ratio 93:7) $\delta 5.95$ (br d, 0.9H, $J=7.7 \mathrm{~Hz}$ ), 5.77 (br d, $0.1 \mathrm{H}, J=7.7 \mathrm{~Hz}$ ), 4.58 (td, $0.9 \mathrm{H}, J=7.8$ and $5.0 \mathrm{~Hz}$ ), 4.35 (dt, $0.1 \mathrm{H}, J=8.1$ and $5.0 \mathrm{~Hz}), 3.77(\mathrm{~s}, 3 \mathrm{H}), 2.52(\mathrm{t}, 2 \mathrm{H}, J=7.3 \mathrm{~Hz}), 2.18(\mathrm{~m}, 1 \mathrm{H}, J=$ 7.5 and $5.0 \mathrm{~Hz}$ ), 2.09 (s, 3H), $1.97(\mathrm{~m}, 1 \mathrm{H}, J=7.2 \mathrm{~Hz}) ;{ }^{13} \mathrm{C} \mathrm{NMR}\left(\mathrm{CDCl}_{3}, 100.62\right.$ $\mathrm{MHz}$ ) (major rotamer) $\delta 171.7,156.3,52.8,52.8,31.3$, 29.8, 15.4; FTIR (neat) $v_{\max }$ 3321 (br), 2145 (br), 1746 (br), 1701 (br), 1529 (br), 1437, 1226 (br) cm ${ }^{-1}$; LRMS (EI) $\mathrm{m} / \mathrm{z}$ (relative intensity) $232\left(\mathrm{M}^{+}, 100\right), 173$ (45), 162 (36), 158 (70), 130 (41), 114 (64), 102 (24), 97 (54), 88 (71), 72 (26), 70 (59); HRMS (EI) calcd for $\mathrm{C}_{7} \mathrm{H}_{12} \mathrm{~N}_{4} \mathrm{O}_{3} \mathrm{~S}\left(\mathrm{M}^{+}\right)$232.0630, found 232.0636.

(L)-Phenylalanine methyl ester carbamoyl azide (2o). Following the general procedure, and starting from $1.00 \mathrm{mmol}$ of $(L)$-phenylalanine methyl ester trifluoroacetate and $2.30 \mathrm{mmol}$ of PhTMG, after purification by flash chromatography (EtOAc/hexanes from 1:9 to 1:4), the product was obtained as a white solid (0.205 g, 84\% yield). $R f: 0.45$ (hex/EtOAc 4:1). Mp: 59.2-59.8 ${ }^{\circ} \mathrm{C}$. [ $\alpha$ ]$_{20}^{\mathrm{D}}+145.08$ (c $\left.1.83, \mathrm{CHCl}_{3}\right) .{ }^{1} \mathrm{H}$ NMR $\left(\mathrm{CDCl}_{3}, 400.13 \mathrm{MHz}\right.$ ) (two rotamers, ratio 9:1) $\delta 7.31-7.21$ (m, 3H), 7.12-7.10 (m, 2H), 5.65 (br d, 0.9H, $J=7.3 \mathrm{~Hz}$ ), 5.45 (br d, $0.1 \mathrm{H}, J=6.6 \mathrm{~Hz}), 4.74(\mathrm{td}, 1 \mathrm{H}, J=8.2$ and $5.9 \mathrm{~Hz}), 4.44(\mathrm{~m}, 0.1 \mathrm{H}), 3.74(\mathrm{~s}, 3 \mathrm{H})$, $3.17\left(\mathrm{dd}, 1 \mathrm{H}, J=14.0\right.$ and $5.6 \mathrm{~Hz}$ ), $3.10\left(\mathrm{dd}, 1 \mathrm{H}, J=14.0\right.$ and $5.6 \mathrm{~Hz}$ ); ${ }^{13} \mathrm{C}$ NMR $\left(\mathrm{CDCl}_{3}, 100.62 \mathrm{MHz}\right)$ (major rotamer) $\delta 171.2,156.0,135.2,129.1,128.7,127.3$, 54.6, 52.5, 37.9; FTIR (KBr) $v_{\max } 3320$ (br), 2178 (br), 2148 (br), 1718 (br), 1700 (br), 1537 (br), 1437, 1303, 1221 (br), 1103, 1022, 940, 758, 742, 702, 659, 558, 490 
$\mathrm{cm}^{-1}$; LRMS (EI) $\mathrm{m} / \mathrm{z}$ (relative intensity) $248\left(\mathrm{M}^{+}\right), 189$ (11), 162 (100), 131 (15), 118 (11), 91 (87), 77 (6); HRMS (EI) calcd for $\mathrm{C}_{11} \mathrm{H}_{12} \mathrm{~N}_{4} \mathrm{O}_{3}\left(\mathrm{M}^{+}\right)$248.0909, found 248.0915 .

(D)-Phenylalanine methyl ester carbamoyl azide (2p). Following the general procedure, and starting from $1.00 \mathrm{mmol}$ of $(L)$-phenylalanine methyl ester trifluoroacetate and $2.30 \mathrm{mmol}$ of PhTMG, after purification by flash chromatography (EtOAc/hexanes from 1:9 to 1:4), the product was obtained as a white solid (0.205 g, 84\% yield). Rf: 0.45 (hex/EtOAc 4:1). [ $\alpha]^{\mathrm{D}}{ }_{20}-142.06$ (c 1.55, $\mathrm{CHCl}_{3}$ ). Spectroscopic data identical to those for $2 \mathbf{0}$.

Compound 4. Following the general procedure and starting from $1.06 \mathrm{mmol}$ of histidine and $2.30 \mathrm{mmol}$ of PhTMG, after purification by flash chromatography (EtOAc/hexanes from 1:1 to 1:0), the product was obtained as a white solid (0.123 g, 65\% yield). Rf 0.70 (MeOH/EtOAc 1:1). [ $\alpha]^{\mathrm{D}}{ }_{20}-37.83$ (с $0.52, \mathrm{CHCl}_{3}$ ). ${ }^{1} \mathrm{H}$ NMR $\left(\mathrm{CDCl}_{3}, 400.13 \mathrm{MHz}\right) \delta 8.18$ (s, 1H), 6.90 (s, 1H), 6.13 (br s, 1H) 4.38 (m, 1H, $J=$ 9.1, 5.2 and $2.1 \mathrm{~Hz}$ ), 3.84 (s, 3H), 3.40 (dd, $1 \mathrm{H}, J=15.7$ and $5.2 \mathrm{~Hz}$ ), 3.15 (m, $1 \mathrm{H}, J$ $=15.7,9.2$ and $1.1 \mathrm{~Hz}) \cdot{ }^{13} \mathrm{C}$ NMR $\left(\mathrm{CD}_{3} \mathrm{OD}, 100.62 \mathrm{MHz}\right) \delta 172.4,150.0,136.0$, 126.7, 126.3, 53.8, 53.5, 23.8. FTIR (KBr) $v_{\max }$ 3223, 3116, 1760, 1718, 1408, 1201 $\mathrm{cm}^{-1}$. LRMS (EI): $\mathrm{m} / \mathrm{z}$ (relative intensity), $195\left(\mathrm{M}^{+}\right.$, 42), 152 (39), 136 (60), 81 (100); HRMS (EI): calcd for $\mathrm{C}_{8} \mathrm{H}_{9} \mathrm{~N}_{3} \mathrm{O}_{3}\left(\mathrm{M}^{+}\right)$195.0644, found 195.0644.

Dibutylcarbamic (diphenyl phosphoric) anhydride (5). DPPA (0.20 mL, 0.93 mmol) was added to an ice-cooled solution of dibutylamine $(0.168 \mathrm{~mL}, 1.0 \mathrm{mmol})$ and PhTMG (0.268 g, $1.4 \mathrm{mmol}$ ) in acetonitrile. $\mathrm{CO}_{2}$ was bubbled through the solution for 30 min until saturation was achieved. The mixture was stirred for $5 \mathrm{~h}$ under a carbon dioxide atmosphere, allowing the temperature to rise to room temperature. The crude product was dissolved in EtOAc and washed with water and with $5 \% \mathrm{HCl}$. The organic phase was dried over anhydrous $\mathrm{Na}_{2} \mathrm{SO}_{4}$, filtered and concentrated under reduced pressure. Compound $\mathbf{5}$ was obtained as a yellowish oil (0.369 g, 98\% yield). $R f 0.33$ (EtOAc/hexanes $1: 5) .{ }^{1} \mathrm{H} \mathrm{NMR}\left(\mathrm{CDCl}_{3}, 400.13 \mathrm{MHz}\right) \delta$ 7.38-7.20 (m, 10H), 3.25 (t, 2H, $J=7.6 \mathrm{~Hz}), 3.08(\mathrm{t}, 2 \mathrm{H}, J=7.6 \mathrm{~Hz}), 1.55(\mathrm{~m}, 2 \mathrm{H})$, 1.43-1.28 (m, 4H), 1.14 (m, 2H, $J=7.4 \mathrm{~Hz}$ ), 0.93 (t, 3H, $J=7.3 \mathrm{~Hz}), 0.85$ (t, 3H, $J=$ $7.3 \mathrm{~Hz}) .{ }^{13} \mathrm{C}$ NMR $\left(\mathrm{CDCl}_{3}, 100.62 \mathrm{MHz}\right) \delta 150.4,150.3,147.8,129.7,125.6,120.3$, 120.3, 48.4, 47.9, 30.5, 29.5, 19.8, 19.7, 13.7, 13.6. ${ }^{31} \mathrm{P} \mathrm{NMR}\left(\mathrm{CDCl}_{3}, 161.97 \mathrm{MHz}\right) \delta$ -1.84. LRMS (EI): $\mathrm{m} / \mathrm{z}$ (relative intensity), $405\left(\mathrm{M}^{+}, 2\right), 349$ (39), 312 (48), 263 (88), 156 (80), 99 (79), 84 (100). HRMS (EI): calcd for $\mathrm{C}_{21} \mathrm{H}_{28} \mathrm{NO}_{5} \mathrm{P}\left(\mathrm{M}^{+}\right)$405.1705, found 405.1716. 
Dibutylcarbamoyl azide (6). Dibutylamine (0.168 mL, $1.0 \mathrm{mmol})$ was submitted to the same procedure as described for the preparation of $\mathbf{5}$. The mixture was heated at $60{ }^{\circ} \mathrm{C}$ for $14 \mathrm{~h}$ under a carbon dioxide atmosphere. The reaction mixture was dissolved in EtOAc, and sequentially washed with water and 5\% $\mathrm{HCl}$. The organic phase was dried over anhydrous $\mathrm{Na}_{2} \mathrm{SO}_{4}$, filtered and concentrated under reduced pressure. The crude product was purified by flash chromatography (EtOAc/hexanes 1:12) to give 6 as a colorless oil (0.095 g, 53\% yield). $R f 0.66$ (EtOAc/hexanes 1:9). ${ }^{1} \mathrm{H}$ NMR $\left(\mathrm{CDCl}_{3}, 400.13 \mathrm{MHz}\right) \delta 3.28(\mathrm{t}, 2 \mathrm{H}, J=7.6 \mathrm{~Hz}), 3.16(\mathrm{t}, 2 \mathrm{H}, J=7.5 \mathrm{~Hz})$, 1.67-1.46 (m, 4H), 1.34-1.26 (m, 4H), $0.93(\mathrm{t}, 6 \mathrm{H}, J=7.3 \mathrm{~Hz}) .{ }^{13} \mathrm{C}$ NMR $\left(\mathrm{CDCl}_{3}\right.$, $100.62 \mathrm{MHz}) \delta 156.3$, 48.7, 47.2, 30.7, 29.8, 20.1, 19.8, 13.8, 13.7. HRMS (FAB+, mNBA matrix): calcd for $\mathrm{C}_{9} \mathrm{H}_{19} \mathrm{~N}_{4} \mathrm{O}(\mathrm{M}+1)^{+} 199.1559$, found 199.1566 . 
${ }^{1} \mathrm{H}$ NMR $\left(\mathrm{CDCl}_{3}\right)$<smiles>N#CC(=O)NCCCCCNC(N)=O</smiles>

$2 a$

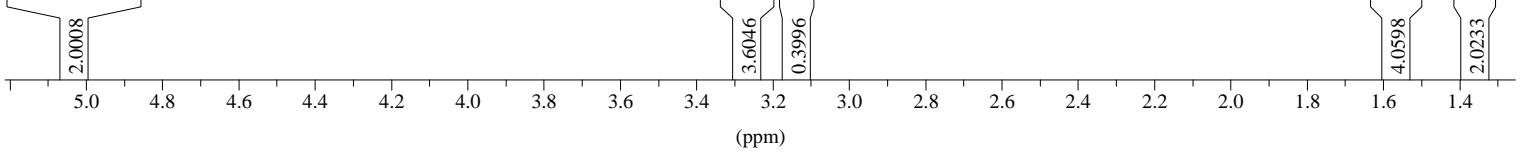

${ }^{13} \mathrm{C}$ NMR $\left(\mathrm{CDCl}_{3}\right)$

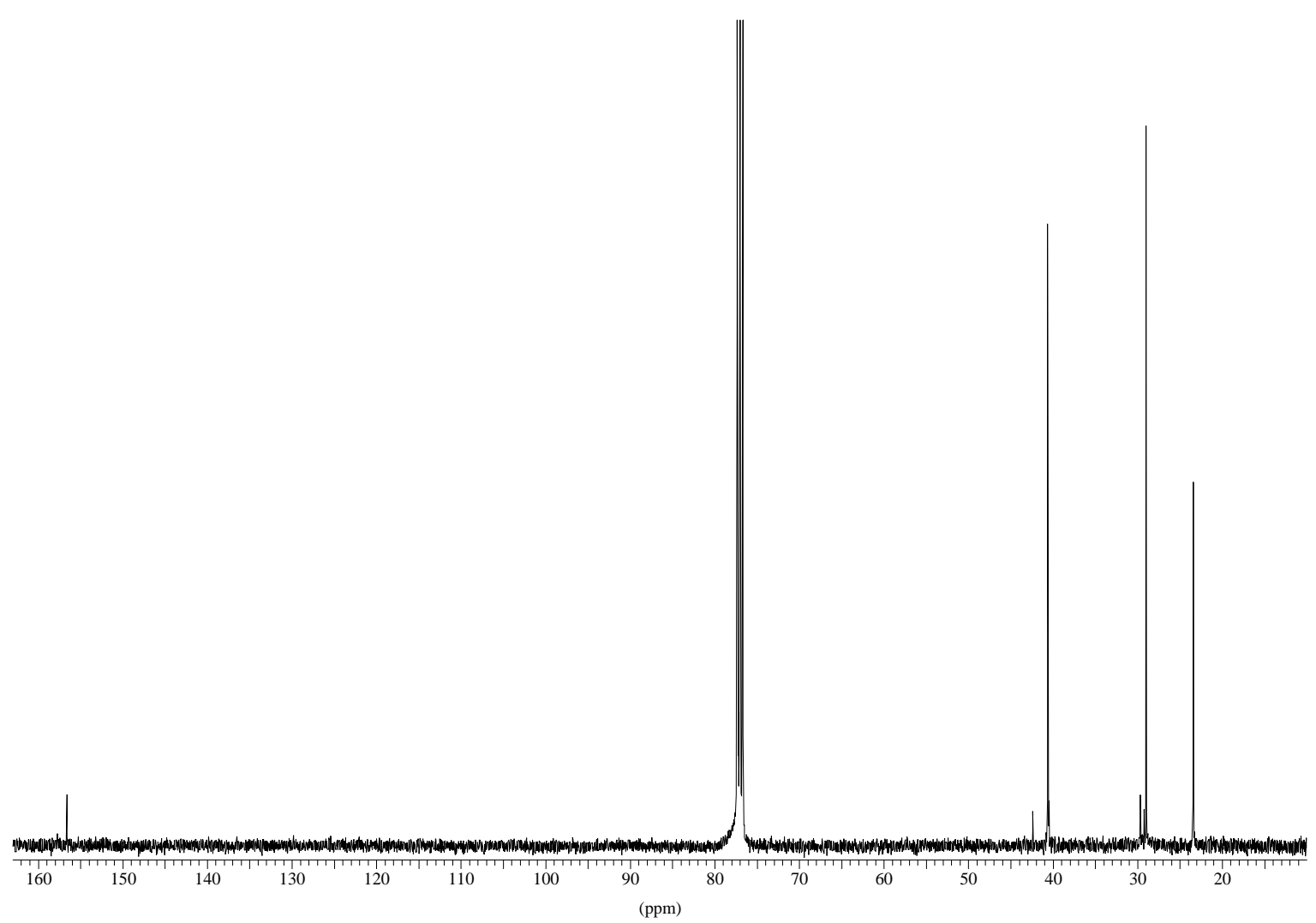


${ }^{1} \mathrm{H}$ NMR $\left(\mathrm{CDCl}_{3}\right)$

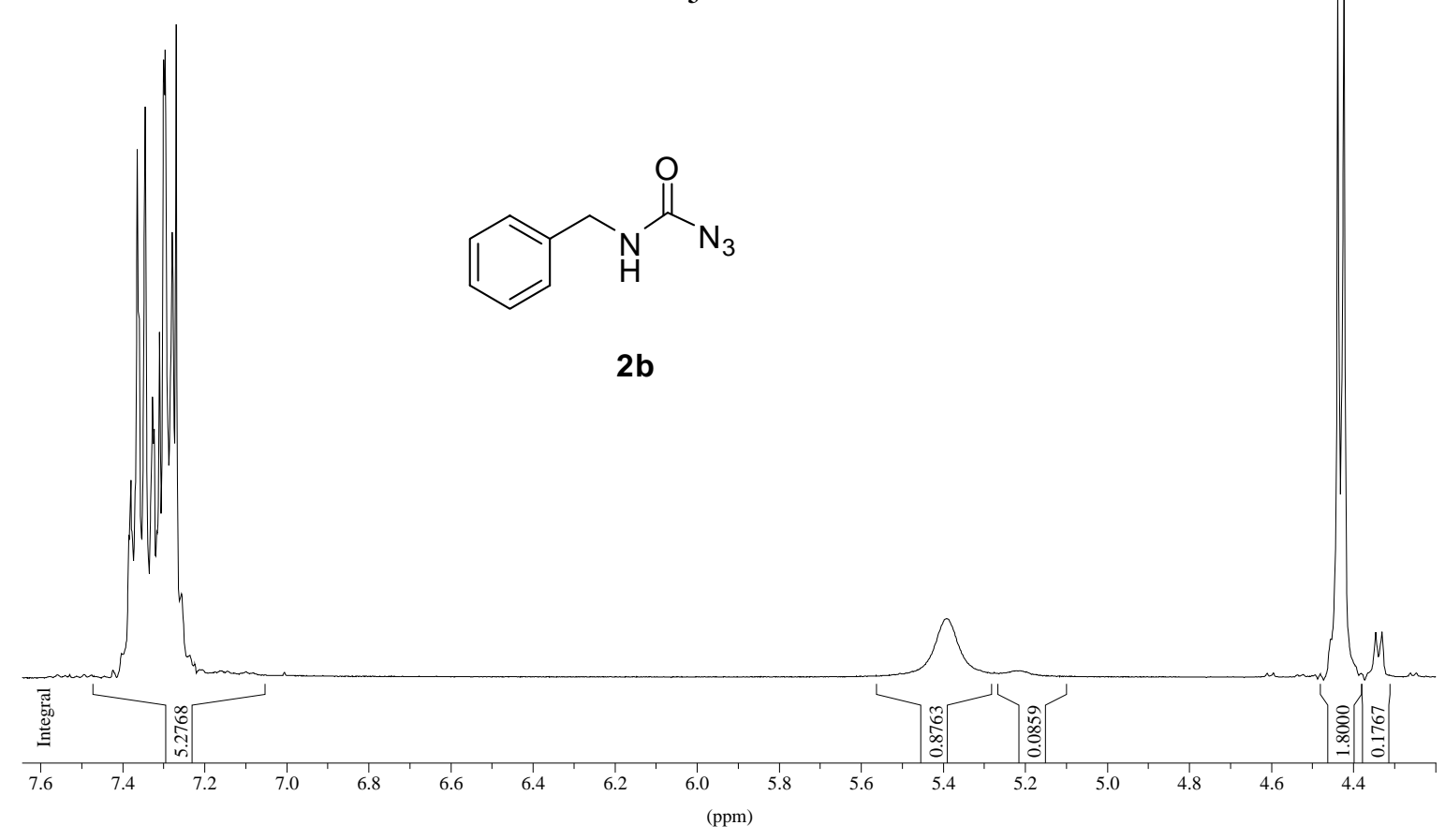

${ }^{13} \mathrm{C}$ NMR $\left(\mathrm{CDCl}_{3}\right)$

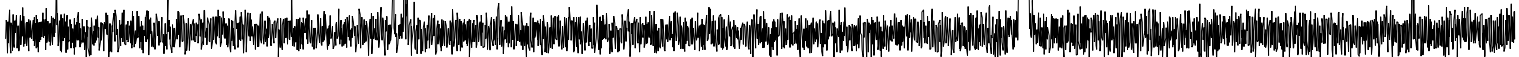

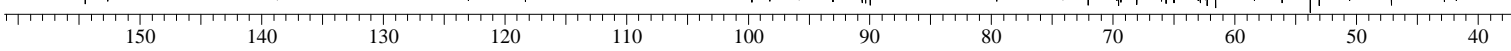
(ppm) 
${ }^{1} \mathrm{H} \operatorname{NMR}\left(\mathrm{CDCl}_{3}\right)$<smiles>[N]C(=O)NCCC1=CCCCC1</smiles>

2c

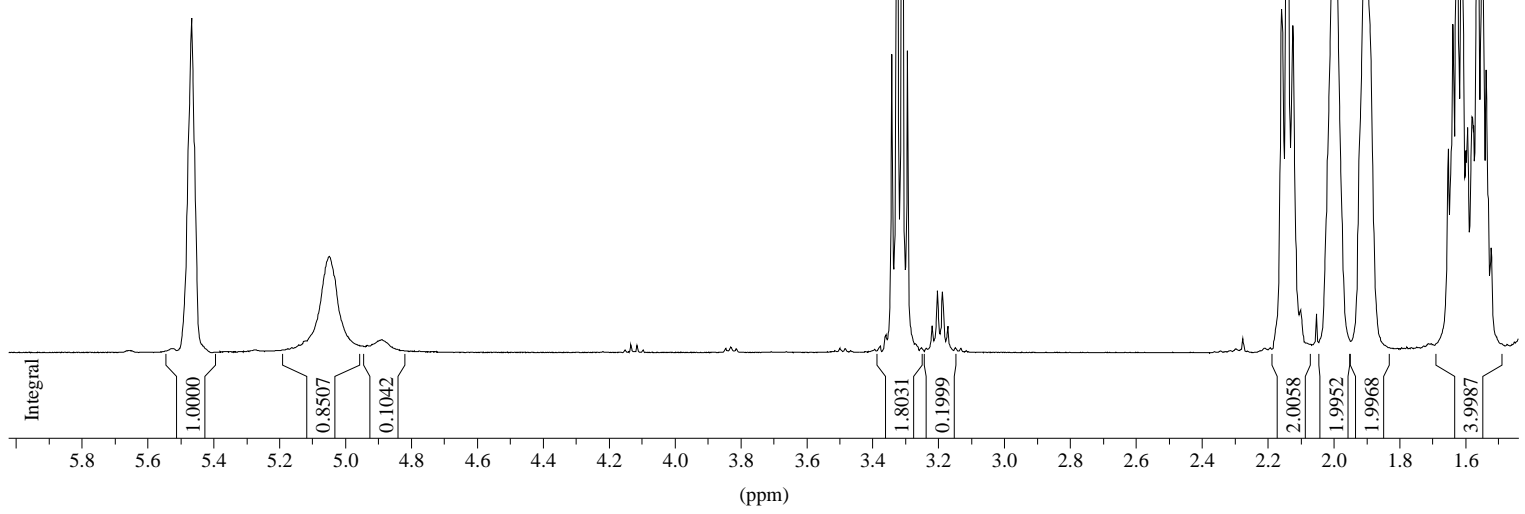

${ }^{13} \mathrm{C} \mathrm{NMR}\left(\mathrm{CDCl}_{3}\right)$

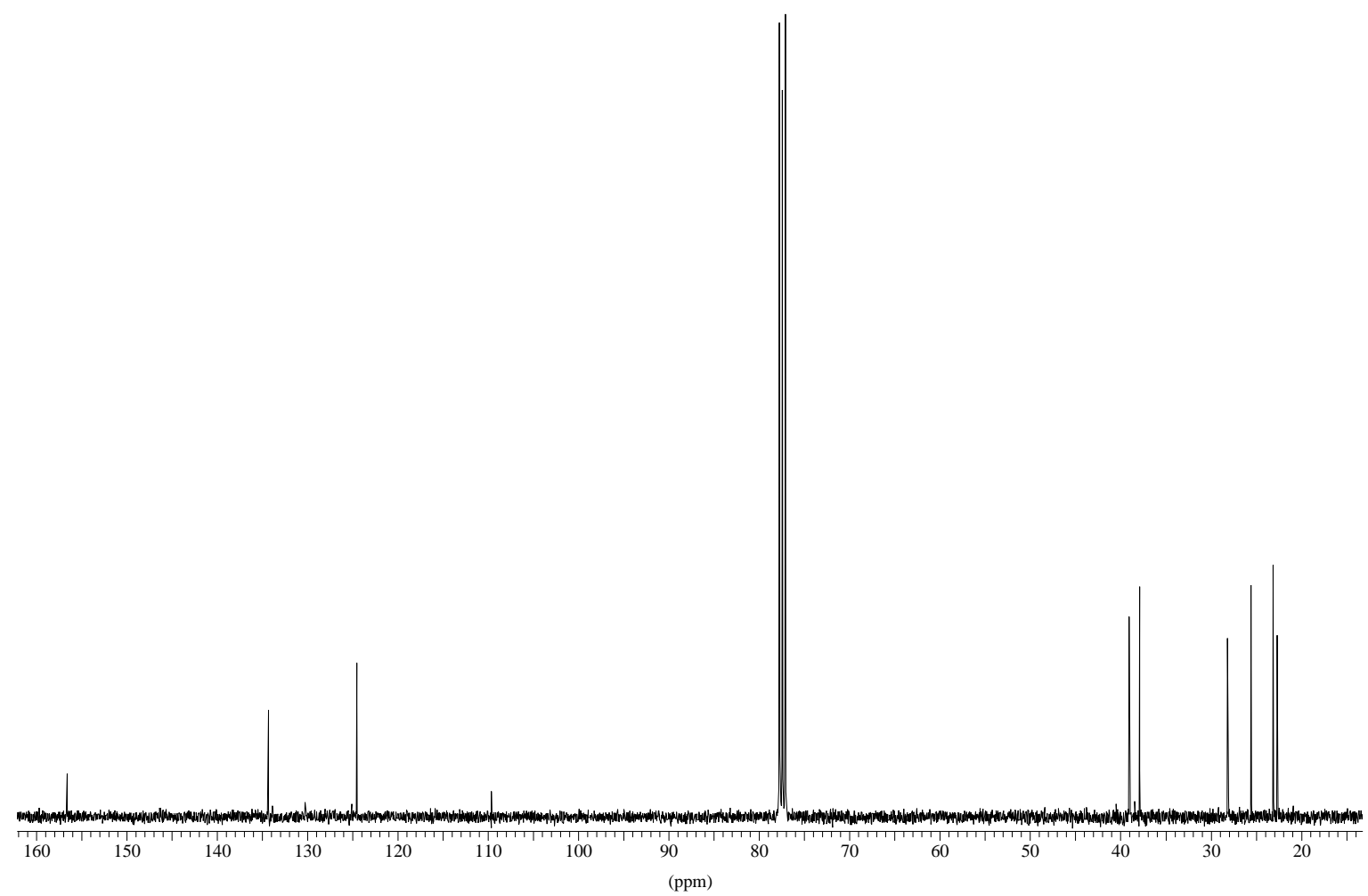




\section{${ }^{1} \mathrm{H}$ NMR $\left(\mathrm{CDCl}_{3}\right)$}<smiles>N#CC(=O)NC1CCCCC1</smiles>

2d

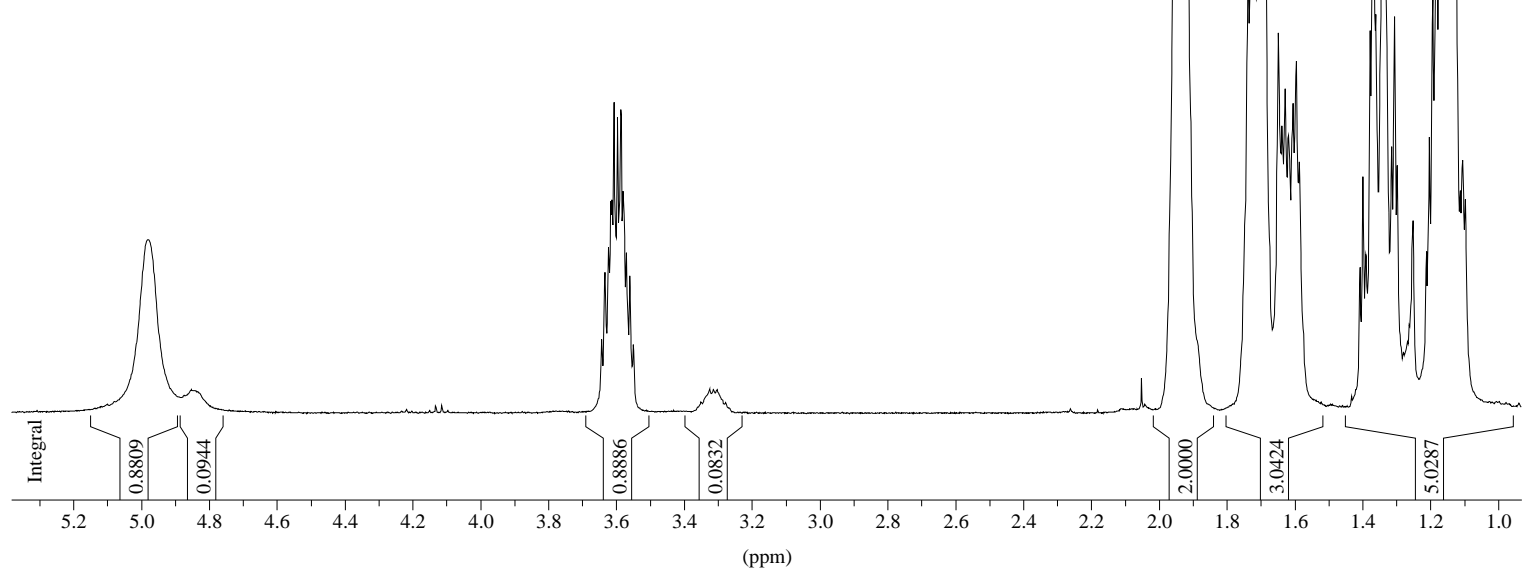

${ }^{13} \mathrm{C}$ NMR $\left(\mathrm{CDCl}_{3}\right)$

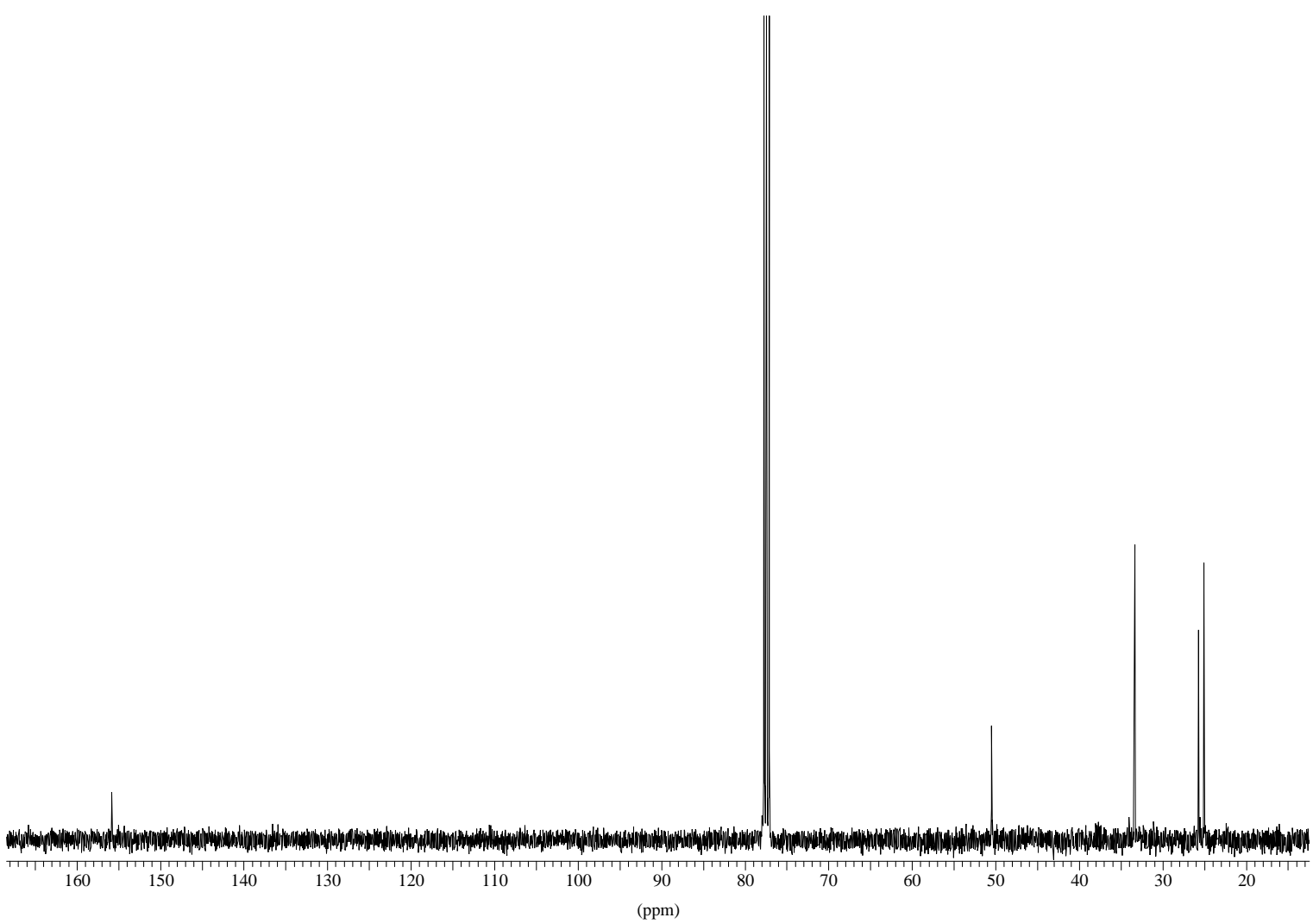




\section{${ }^{1} \mathrm{H}$ NMR $\left(\mathrm{CDCl}_{3}\right)$}

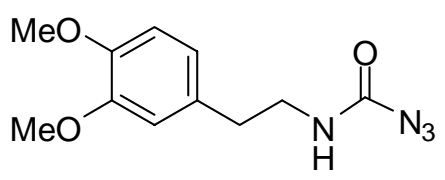

$2 e$

\section{${ }^{13} \mathrm{C} \mathrm{NMR}\left(\mathrm{CDCl}_{3}\right)$}

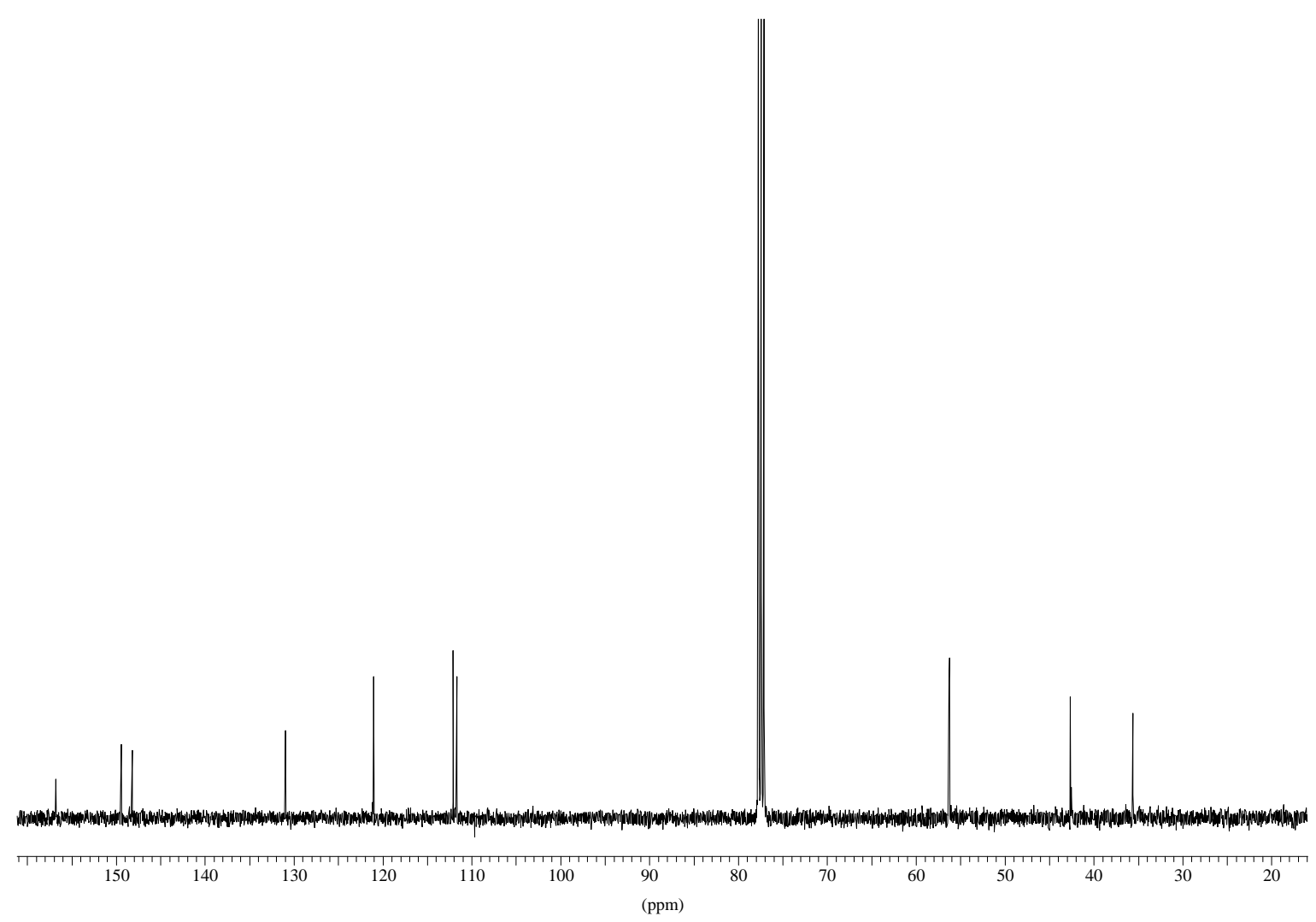




\section{${ }^{1} \mathrm{H}$ NMR $\left(\mathrm{CDCl}_{3}\right)$}

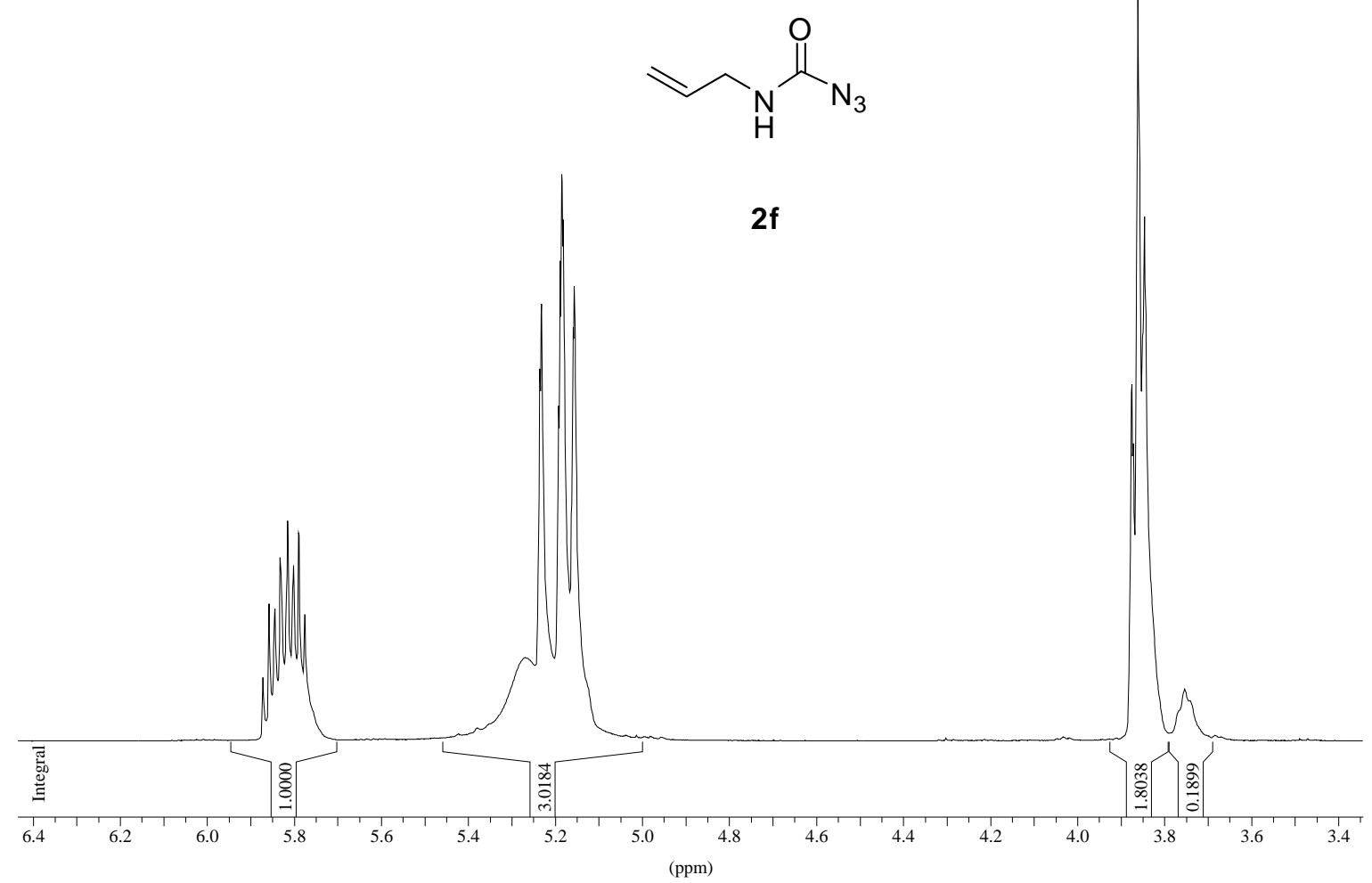

${ }^{13} \mathrm{C} \mathrm{NMR}\left(\mathrm{CDCl}_{3}\right)$

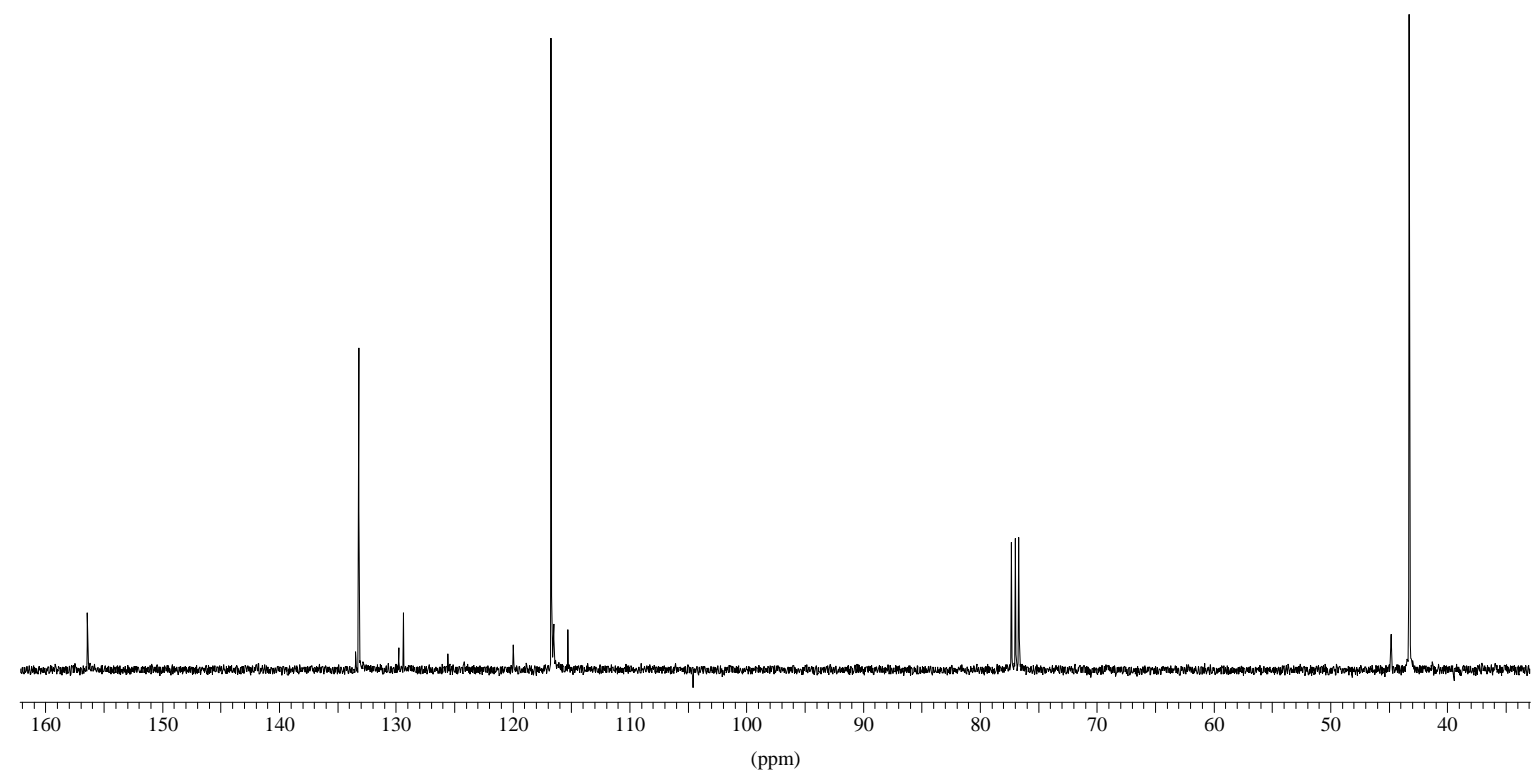


${ }^{1} \mathrm{H}$ NMR $\left(\mathrm{CDCl}_{3}\right)$<smiles>CC(C)CNC(N)=O</smiles>

$2 g$

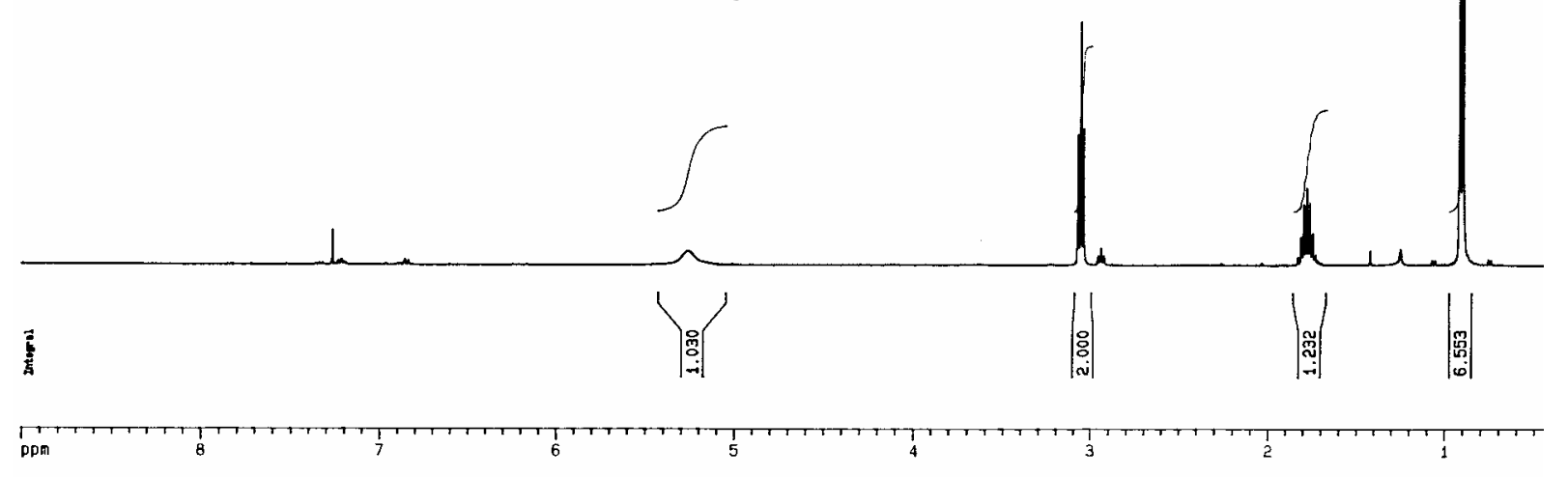

${ }^{13} \mathrm{C}$ NMR $\left(\mathrm{CDCl}_{3}\right)$

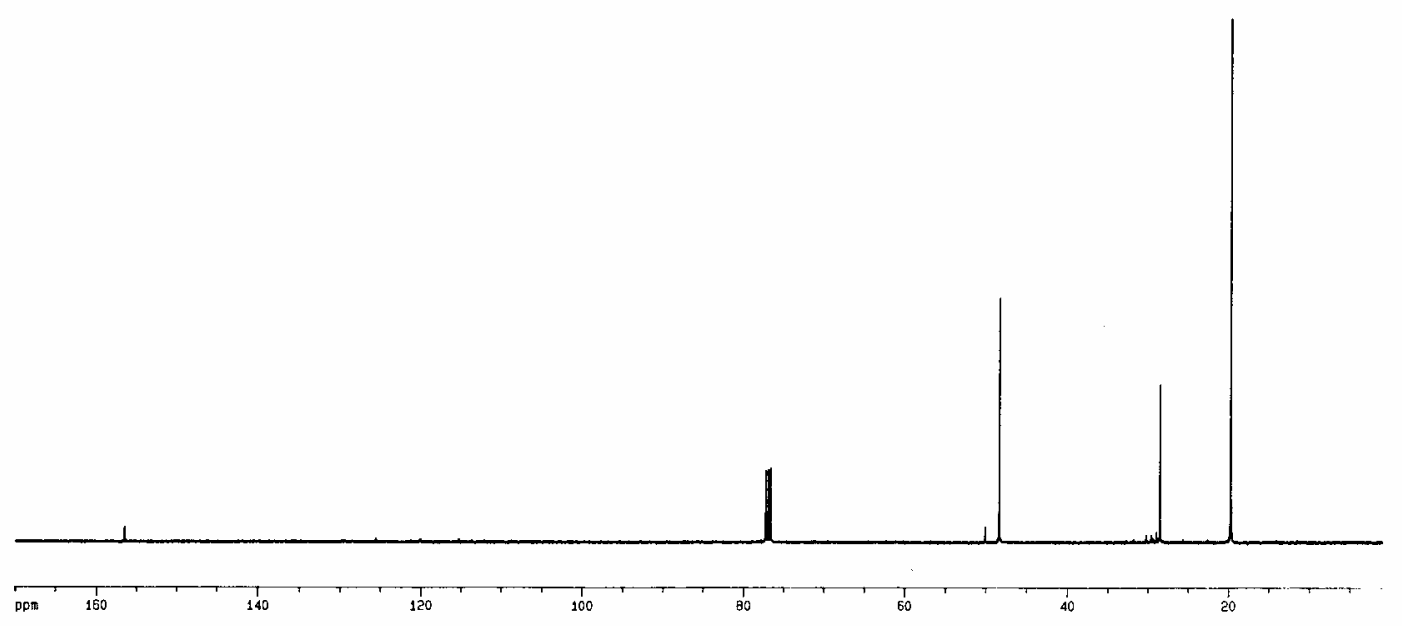




\section{${ }^{1} \mathrm{H}$ NMR $\left(\mathrm{CDCl}_{3}\right)$}<smiles>COC(=O)C(NC(N)=O)C(C)C</smiles>

$2 h$

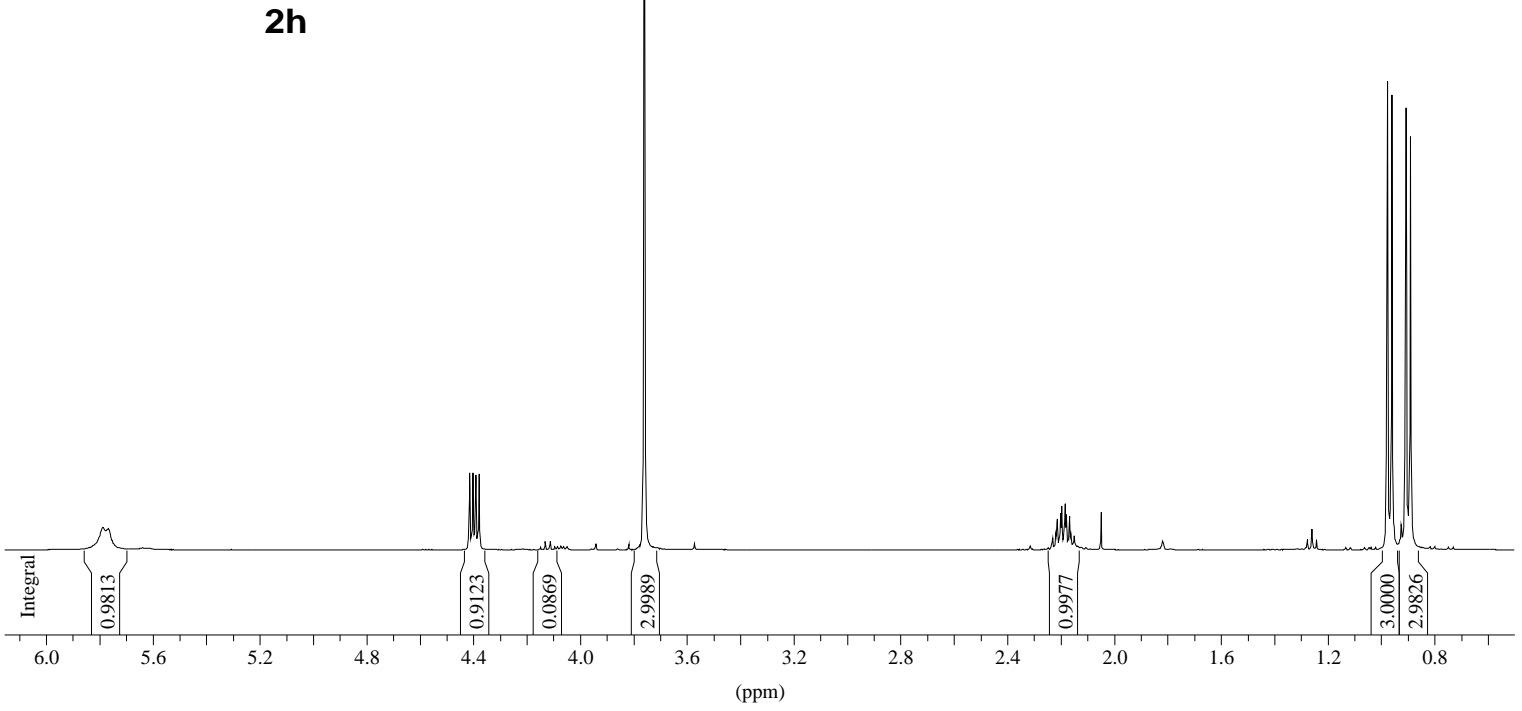

${ }^{13} \mathrm{C} \mathrm{NMR}\left(\mathrm{CDCl}_{3}\right)$

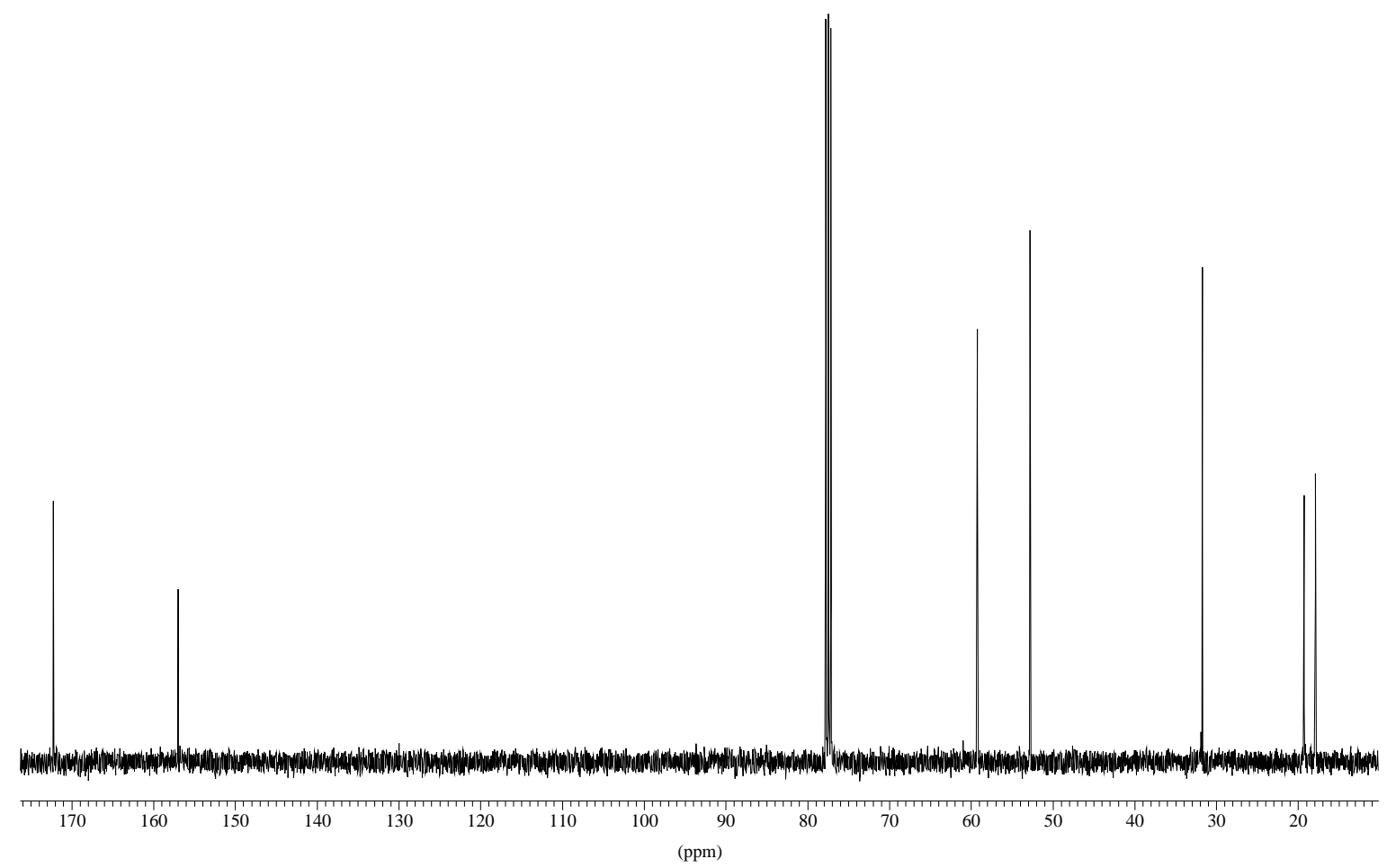


${ }^{1} \mathrm{H}$ NMR $\left(\mathrm{CDCl}_{3}\right)$<smiles>COC(=O)[C@H](NC(N)=O)C(C)C</smiles>

$2 \mathrm{i}$

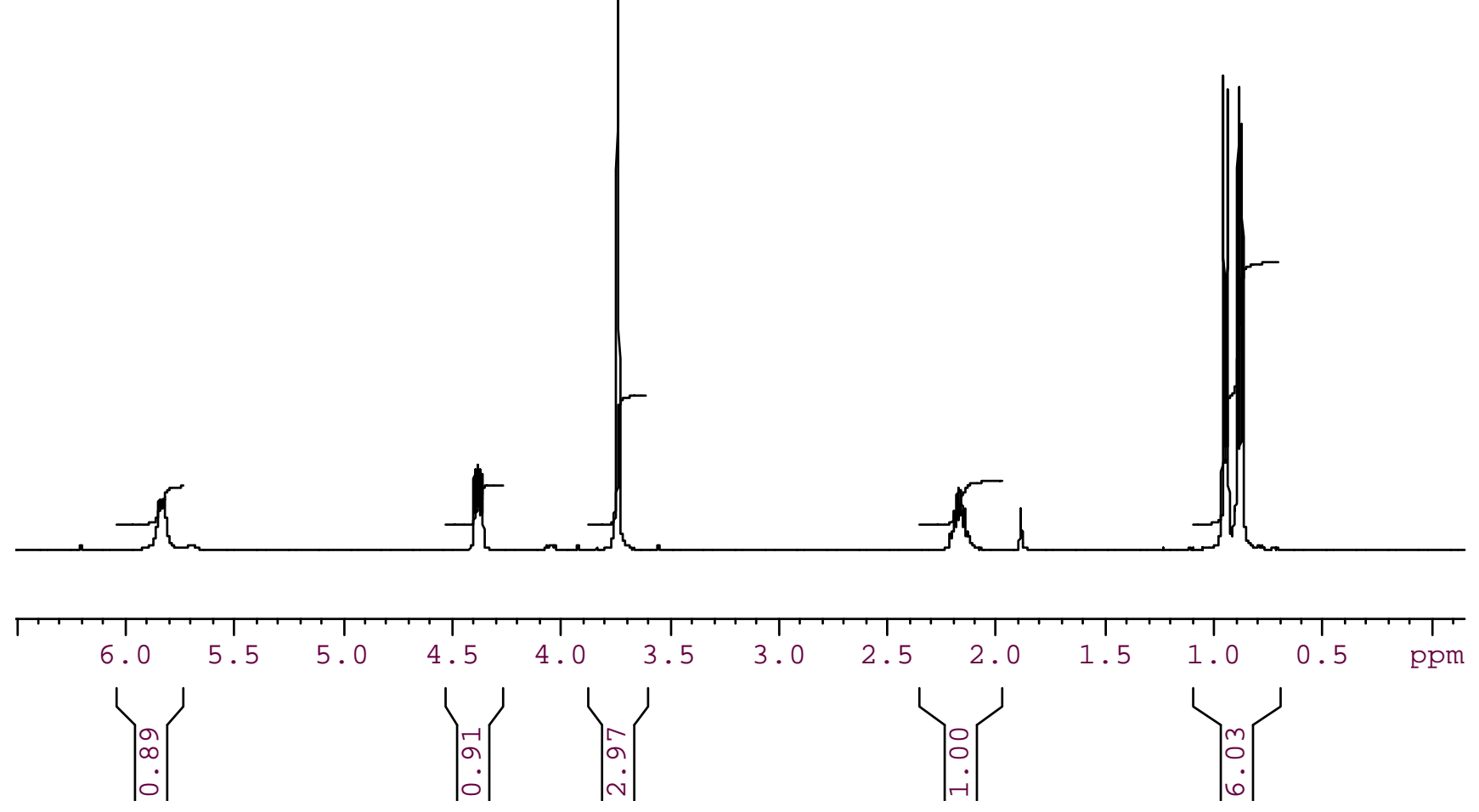

${ }^{13} \mathrm{C}$ NMR $\left(\mathrm{CDCl}_{3}\right)$

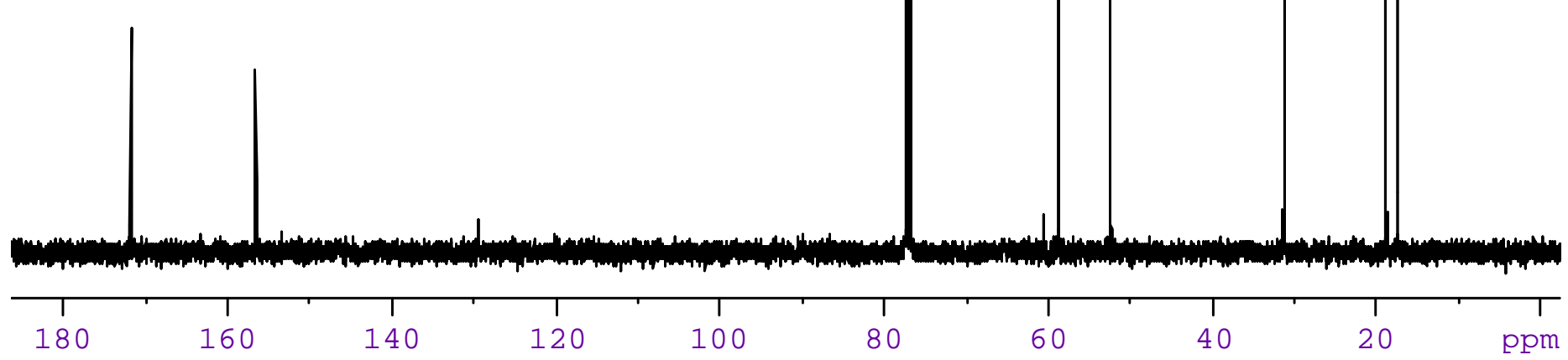


${ }^{1} \mathrm{H}$ NMR $\left(\mathrm{CDCl}_{3}\right)$<smiles>COC(=O)CC[C@H](NC(N)=O)C(=O)OC</smiles>

2j

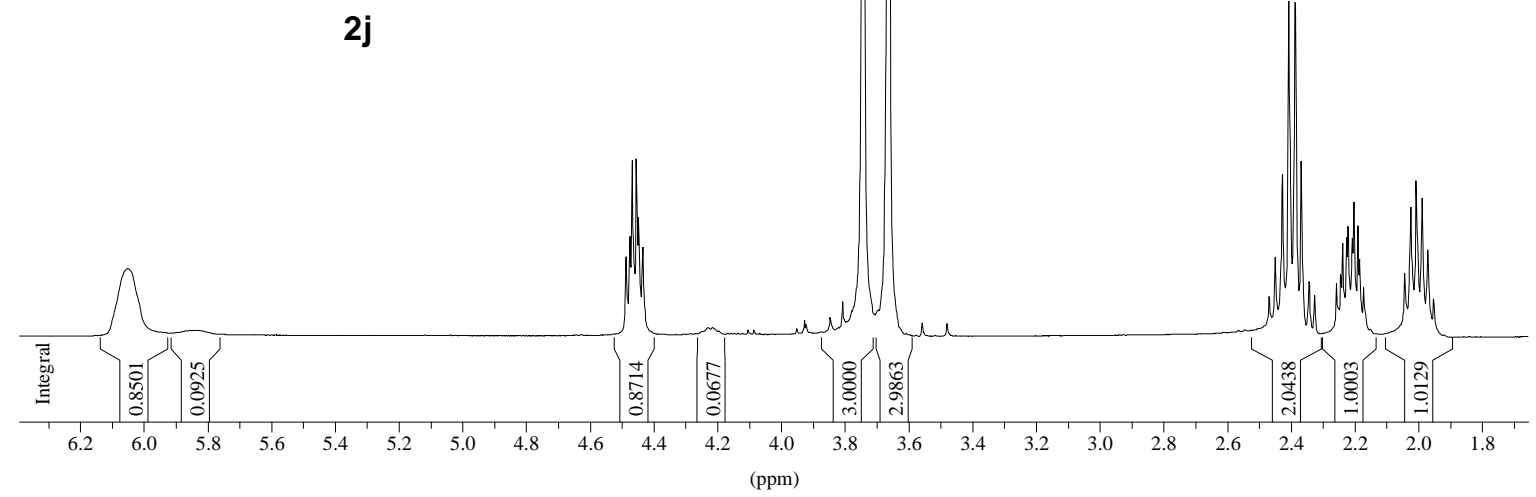

${ }^{13} \mathrm{C}$ NMR $\left(\mathrm{CDCl}_{3}\right)$

DEPT
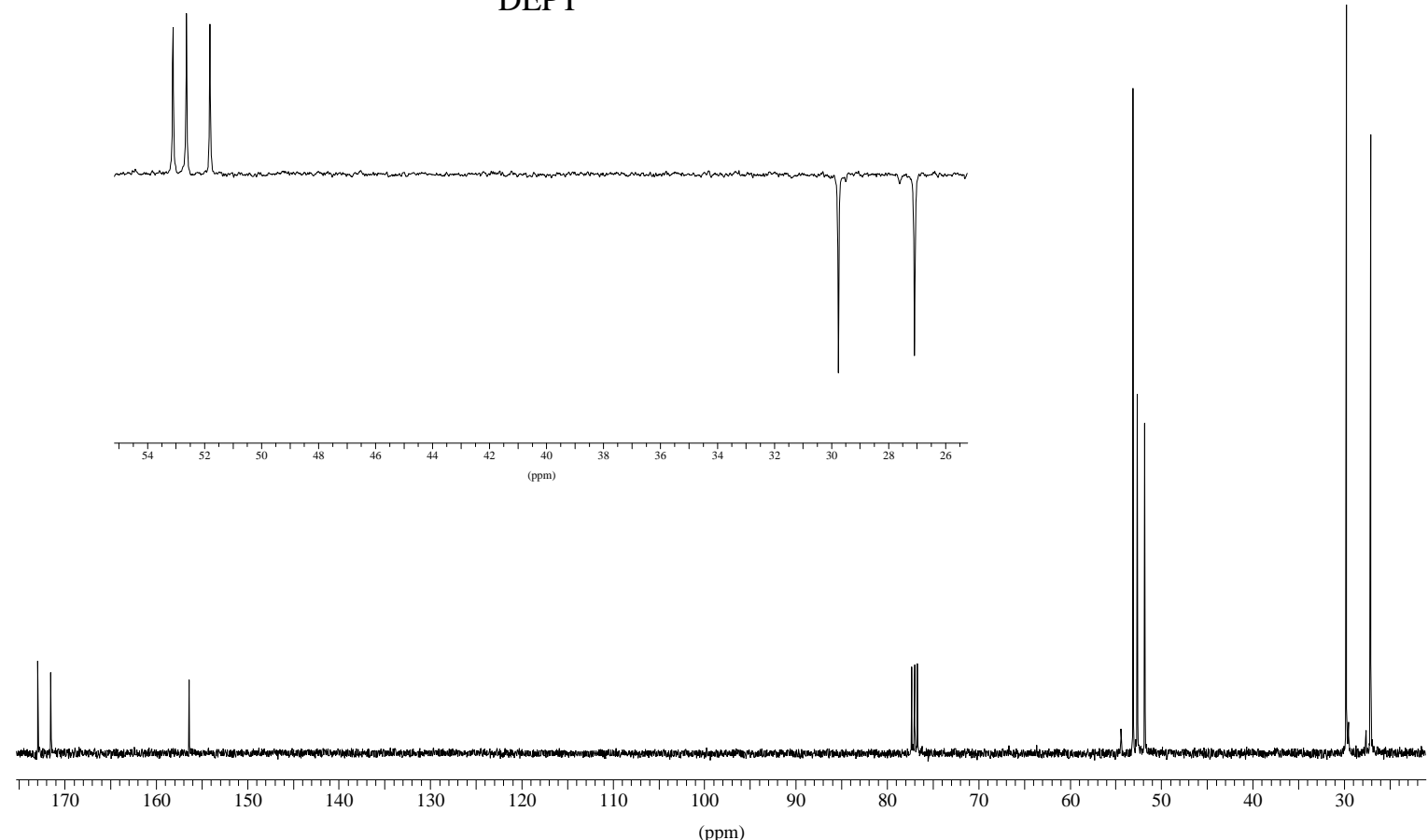
${ }^{1} \mathrm{H}$ NMR $\left(\mathrm{CDCl}_{3}\right)$

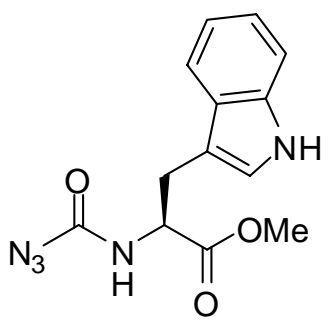

$2 k$

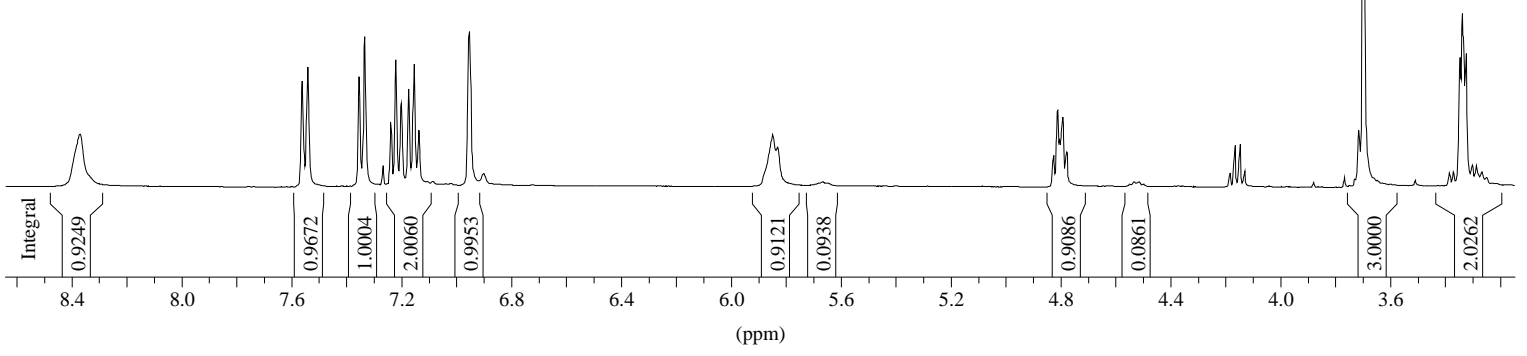

${ }^{13} \mathrm{C} \mathrm{NMR}\left(\mathrm{CDCl}_{3}\right)$

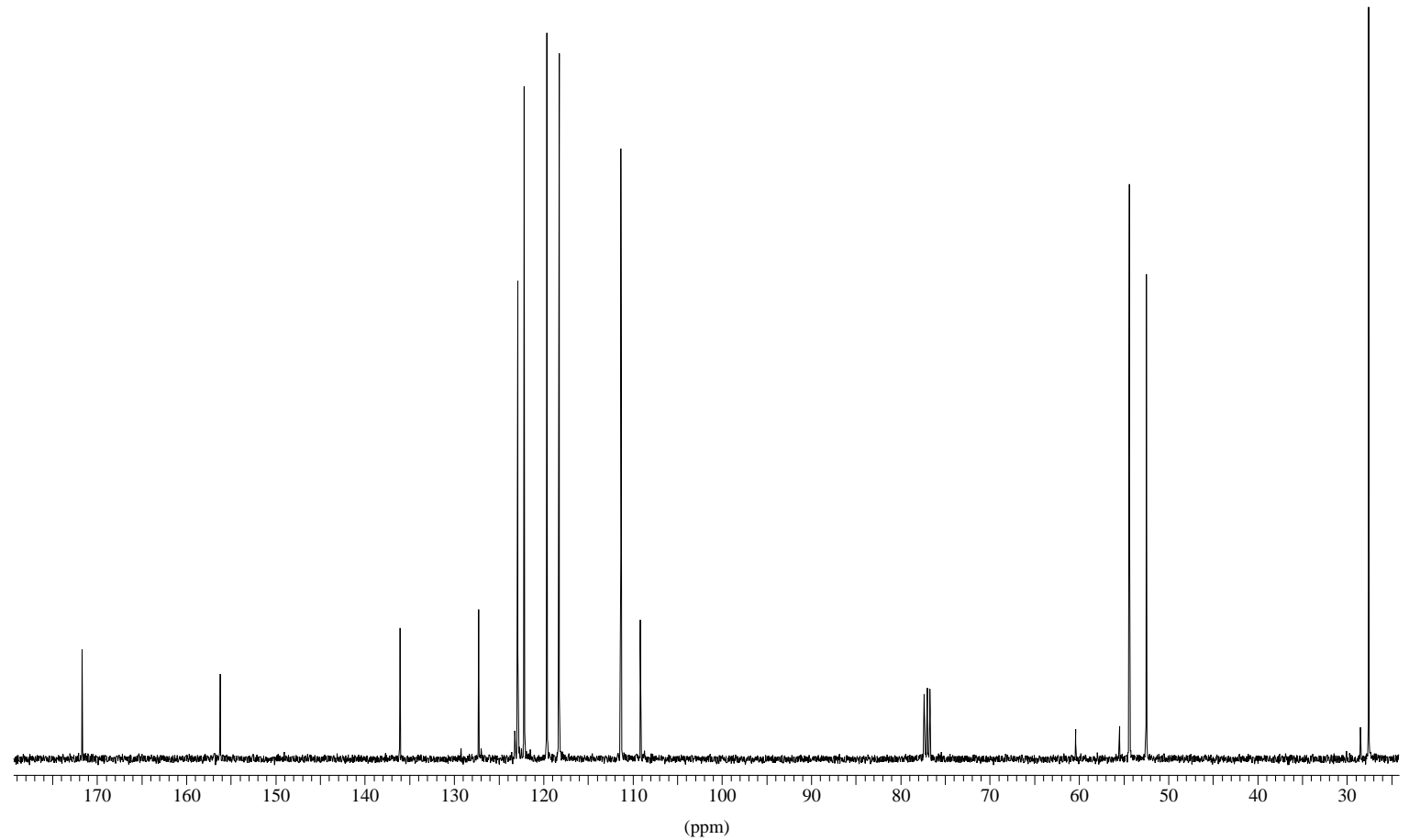


${ }^{1} \mathrm{H}$ NMR $\left(\mathrm{CDCl}_{3}\right)$

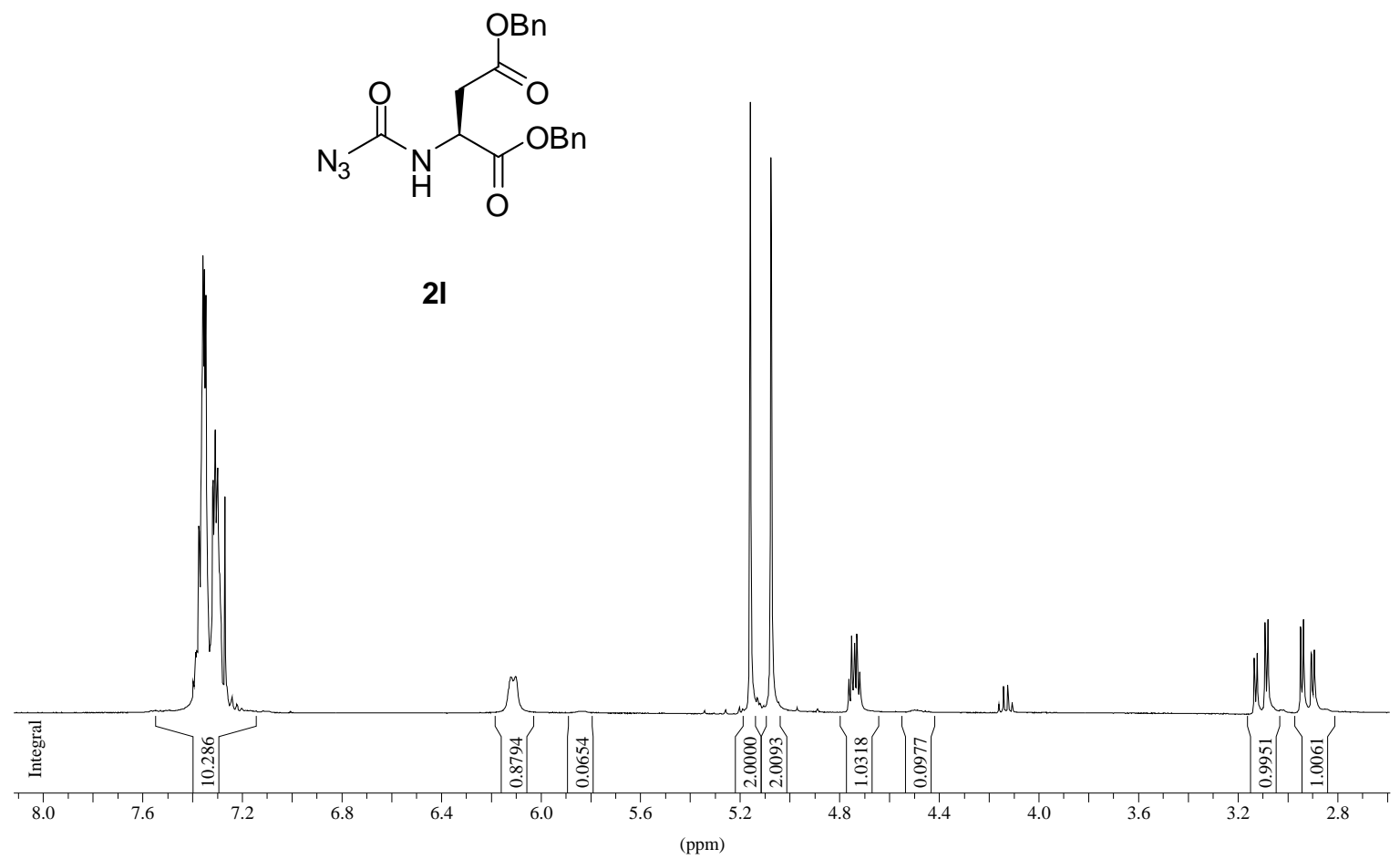

${ }^{13} \mathrm{C} \mathrm{NMR}\left(\mathrm{CDCl}_{3}\right)$

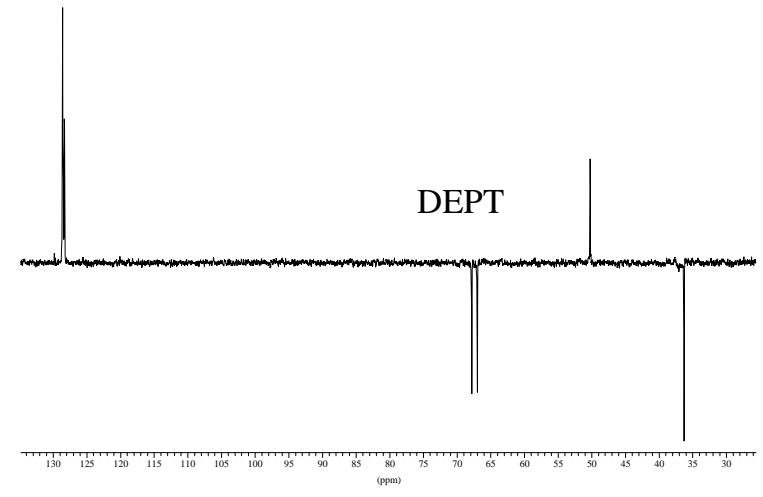

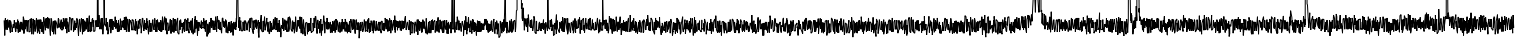
$\begin{array}{llllllllllllll}170 & 160 & 150 & 140 & 130 & 120 & 110 & 100 & 90 & 80 & 70 & 60 & 50 & 40\end{array}$ 
${ }^{1} \mathrm{H}$ NMR $\left(\mathrm{CDCl}_{3}\right)$
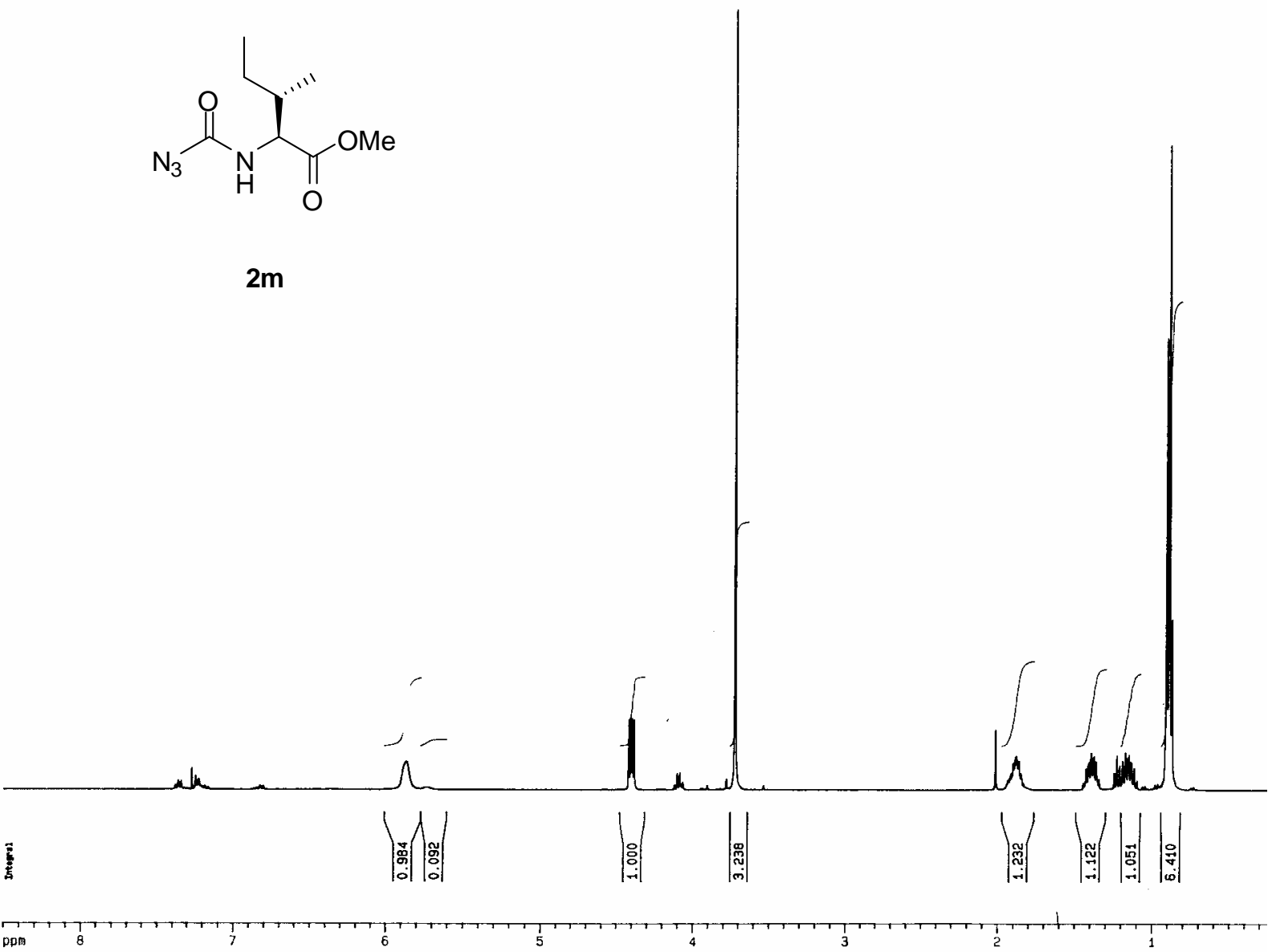

${ }^{13} \mathrm{C} \mathrm{NMR}\left(\mathrm{CDCl}_{3}\right)$

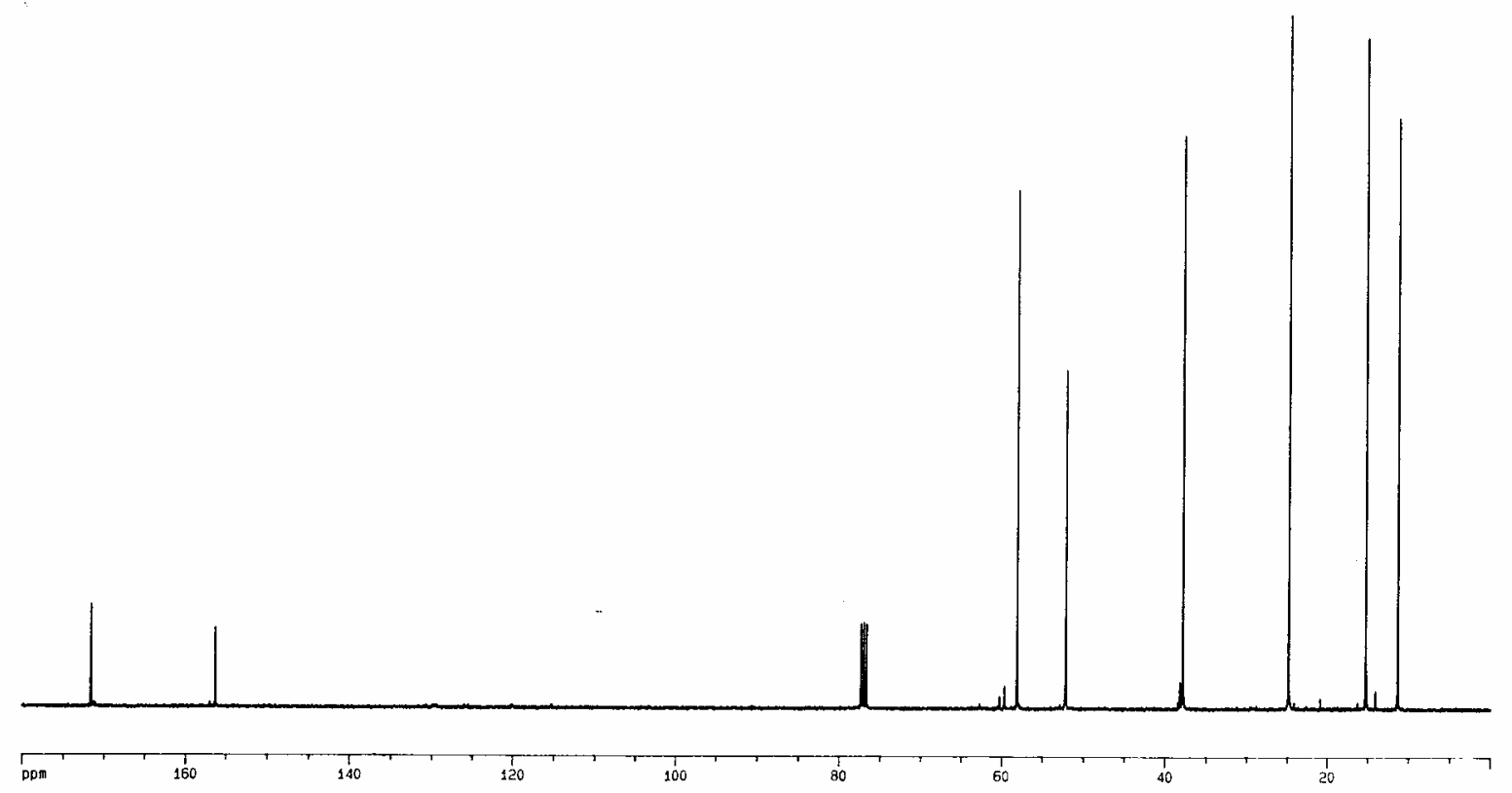


${ }^{1} \mathrm{H}$ NMR ( $\left.\mathrm{CDCl}_{3}\right)$<smiles>COC(=O)C(CCSC)NC(N)=O</smiles>

$2 n$

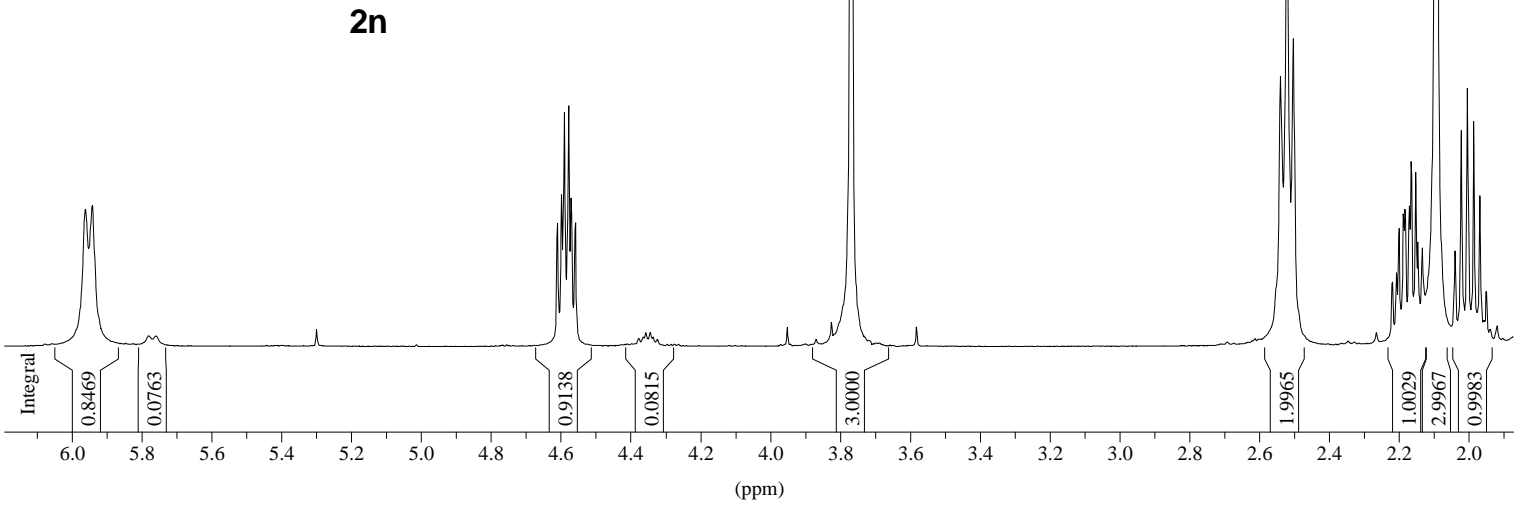

${ }^{13} \mathrm{C}$ NMR $\left(\mathrm{CDCl}_{3}\right)$

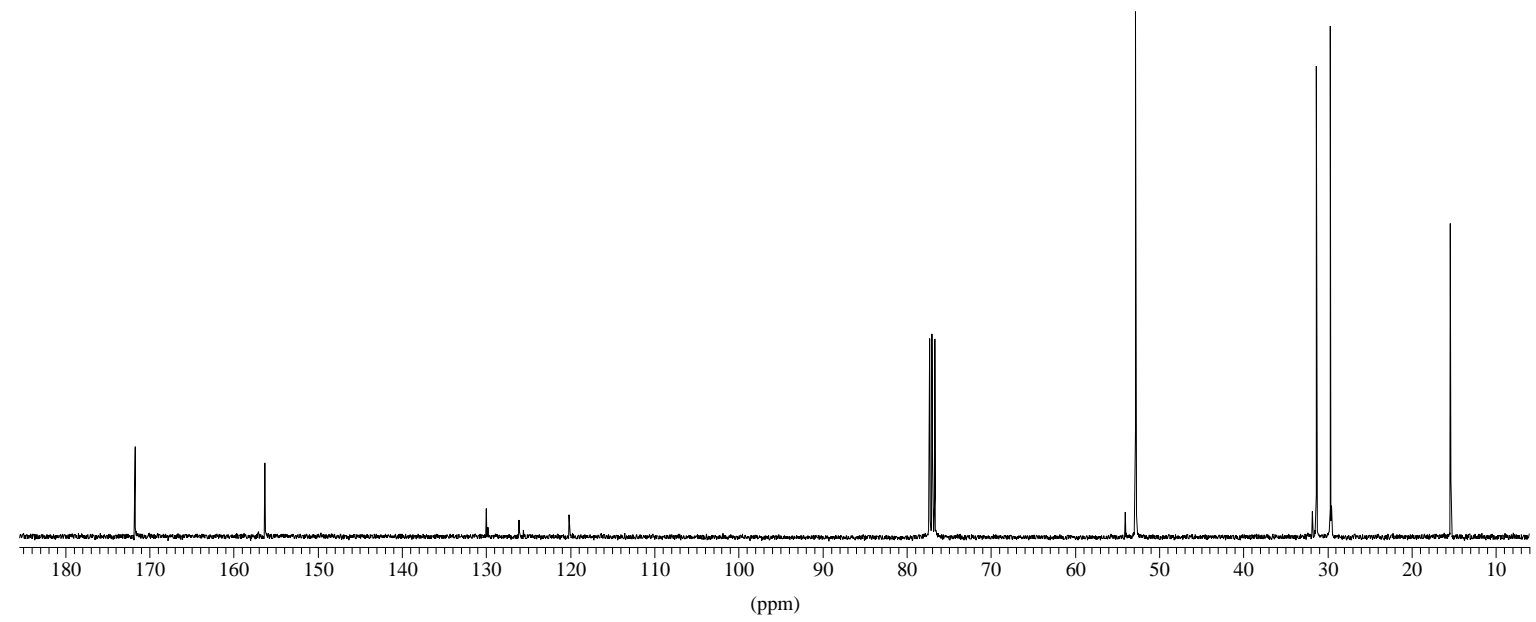


${ }^{1} \mathrm{H}$ NMR ( $\left.\mathrm{CDCl}_{3}\right)$<smiles>COC(=O)C(Cc1ccccc1)NC(N)=O</smiles>

20

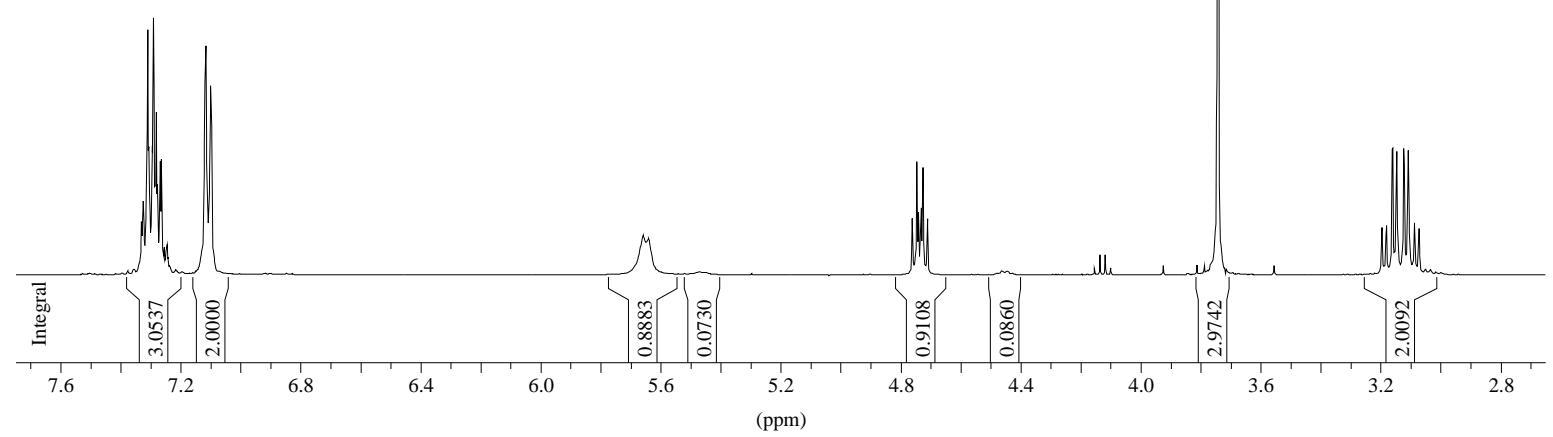

${ }^{13} \mathrm{C}$ NMR $\left(\mathrm{CDCl}_{3}\right)$

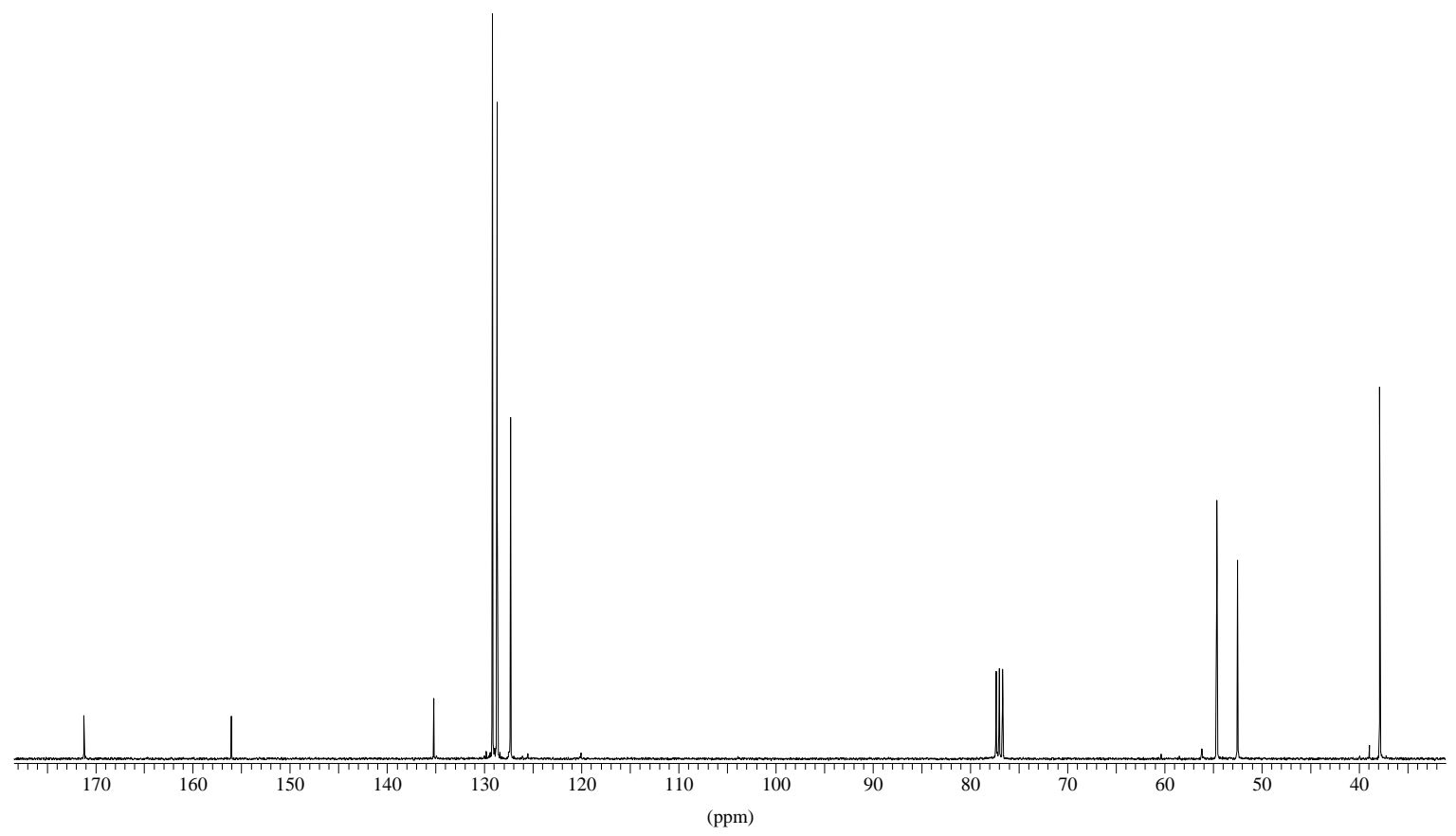




\section{${ }^{1} \mathrm{H}$ NMR $\left(\mathrm{CDCl}_{3}\right)$}

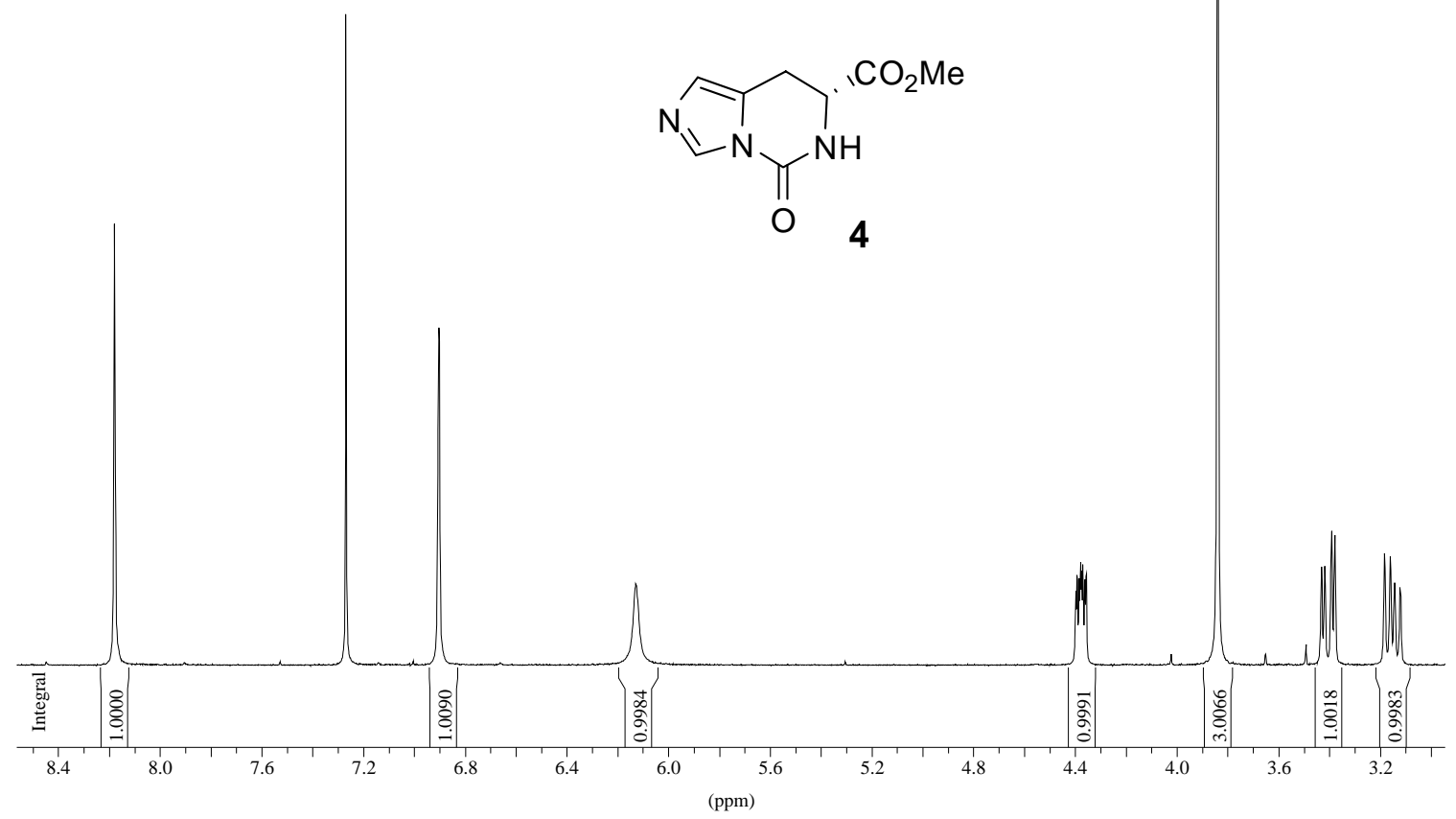

${ }^{13} \mathrm{C}$ NMR (MeOH-d $)$

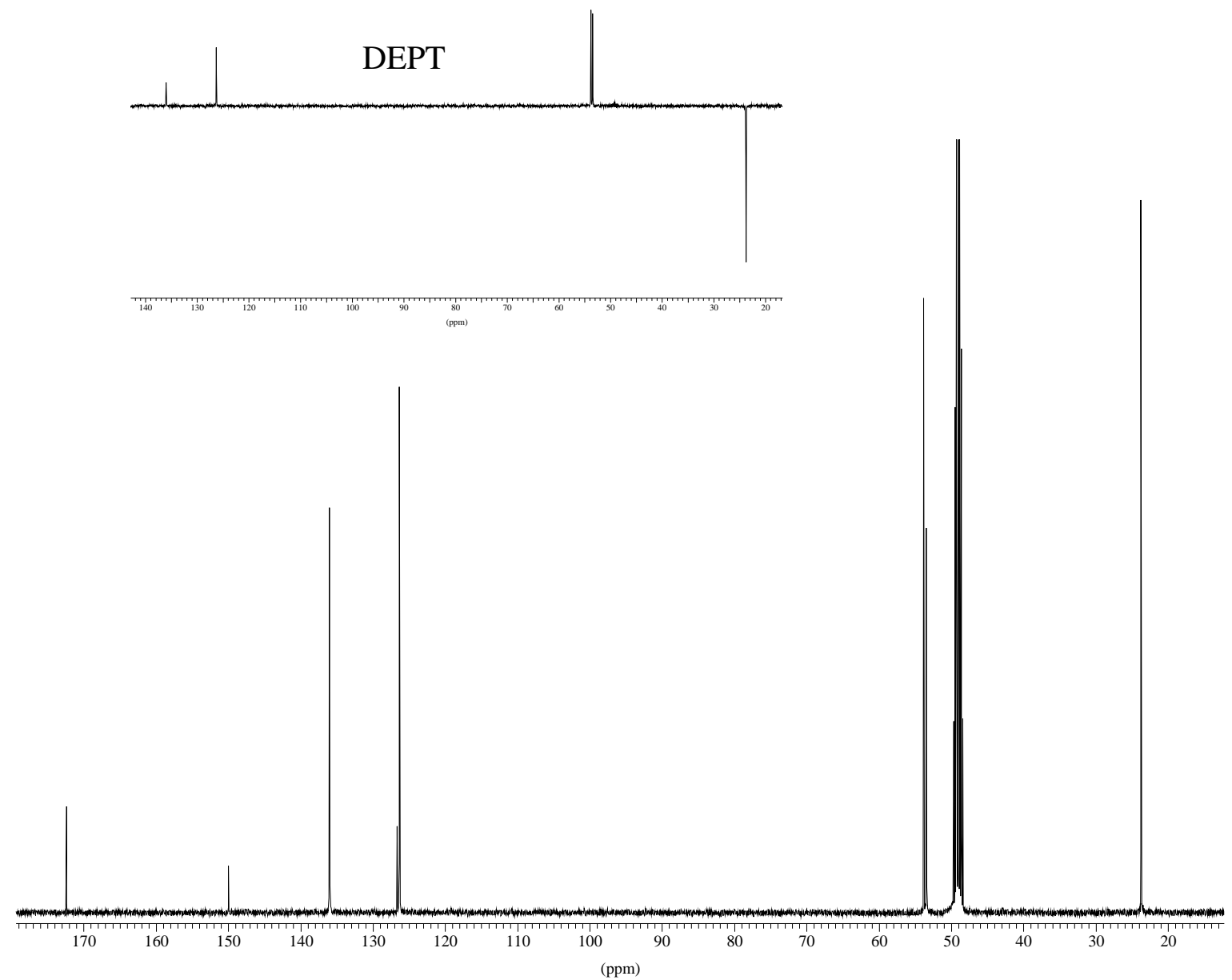




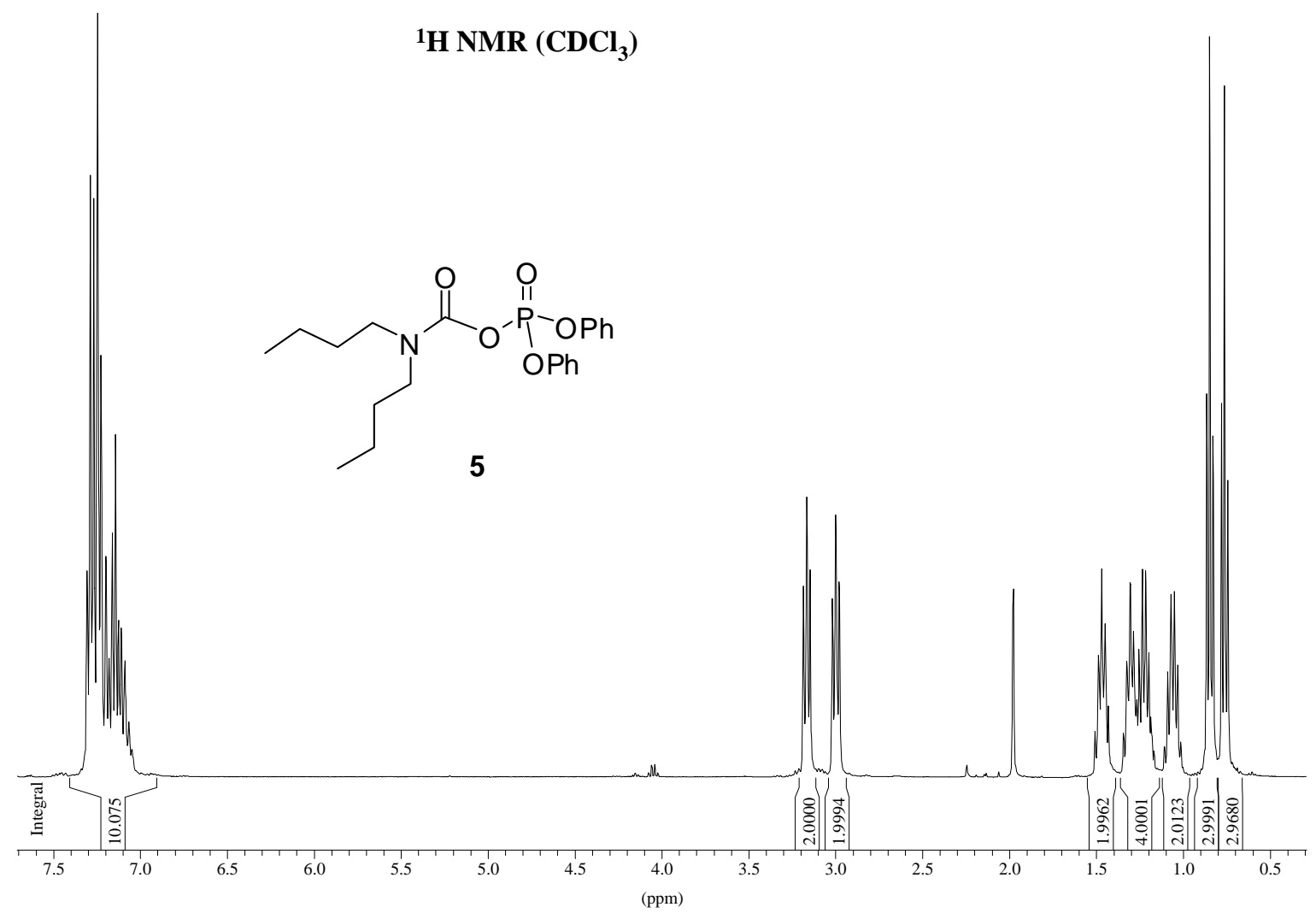

${ }^{13} \mathrm{C}$ NMR $\left(\mathrm{CDCl}_{3}\right)$

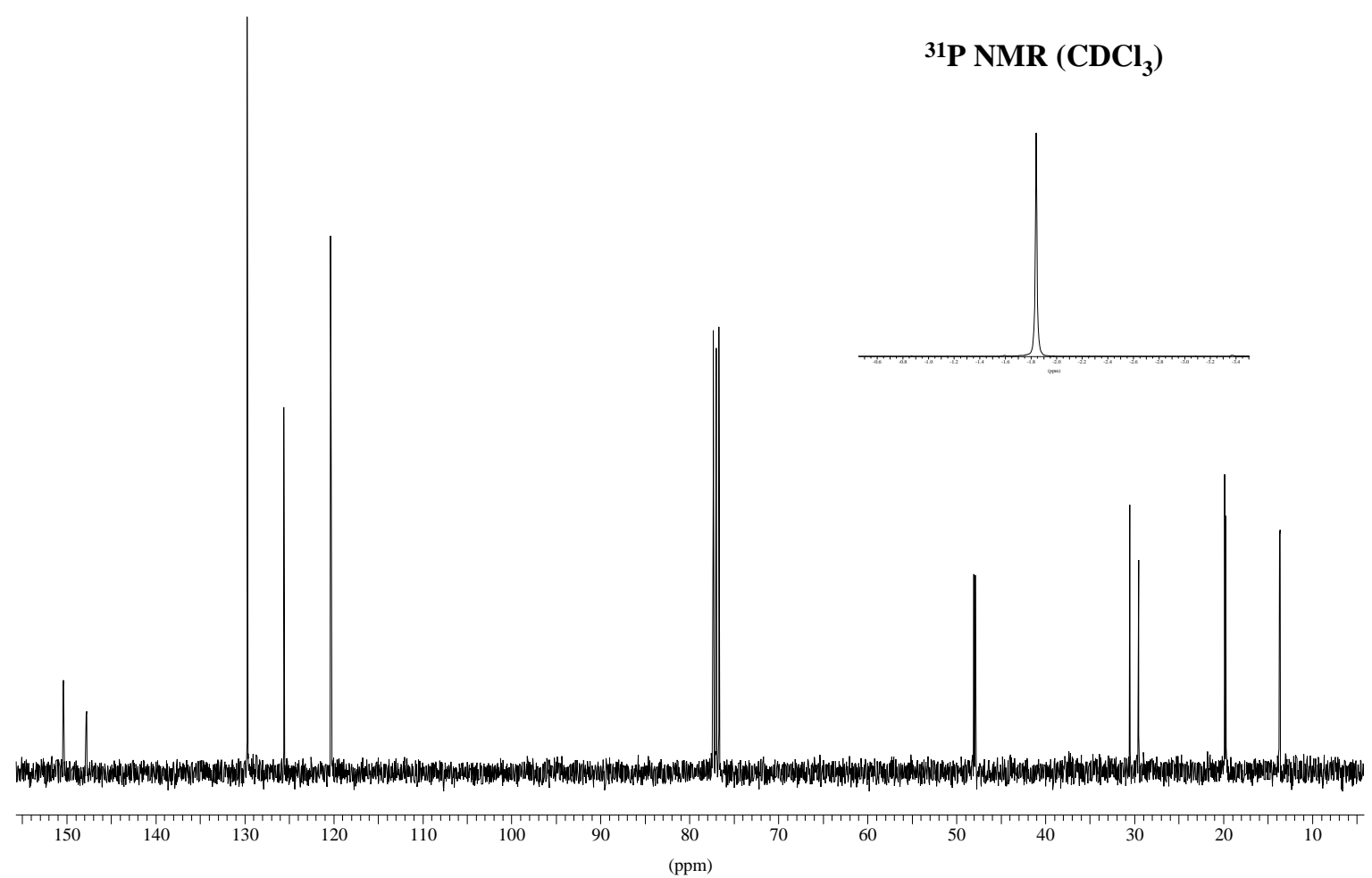


${ }^{1} \mathrm{H}$ NMR (CDCl $\left.{ }_{3}\right)$<smiles>CCCCN(CCCC)C(N)=O</smiles>

6
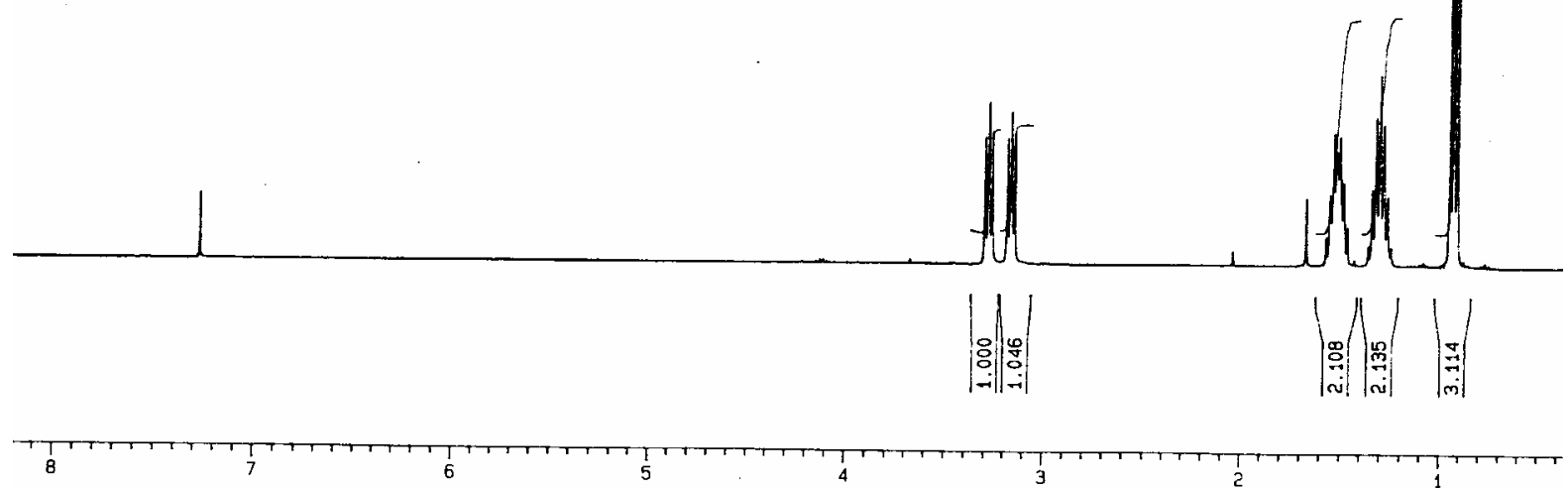

${ }^{13} \mathrm{C}$ NMR $\left(\mathrm{CDCl}_{3}\right)$

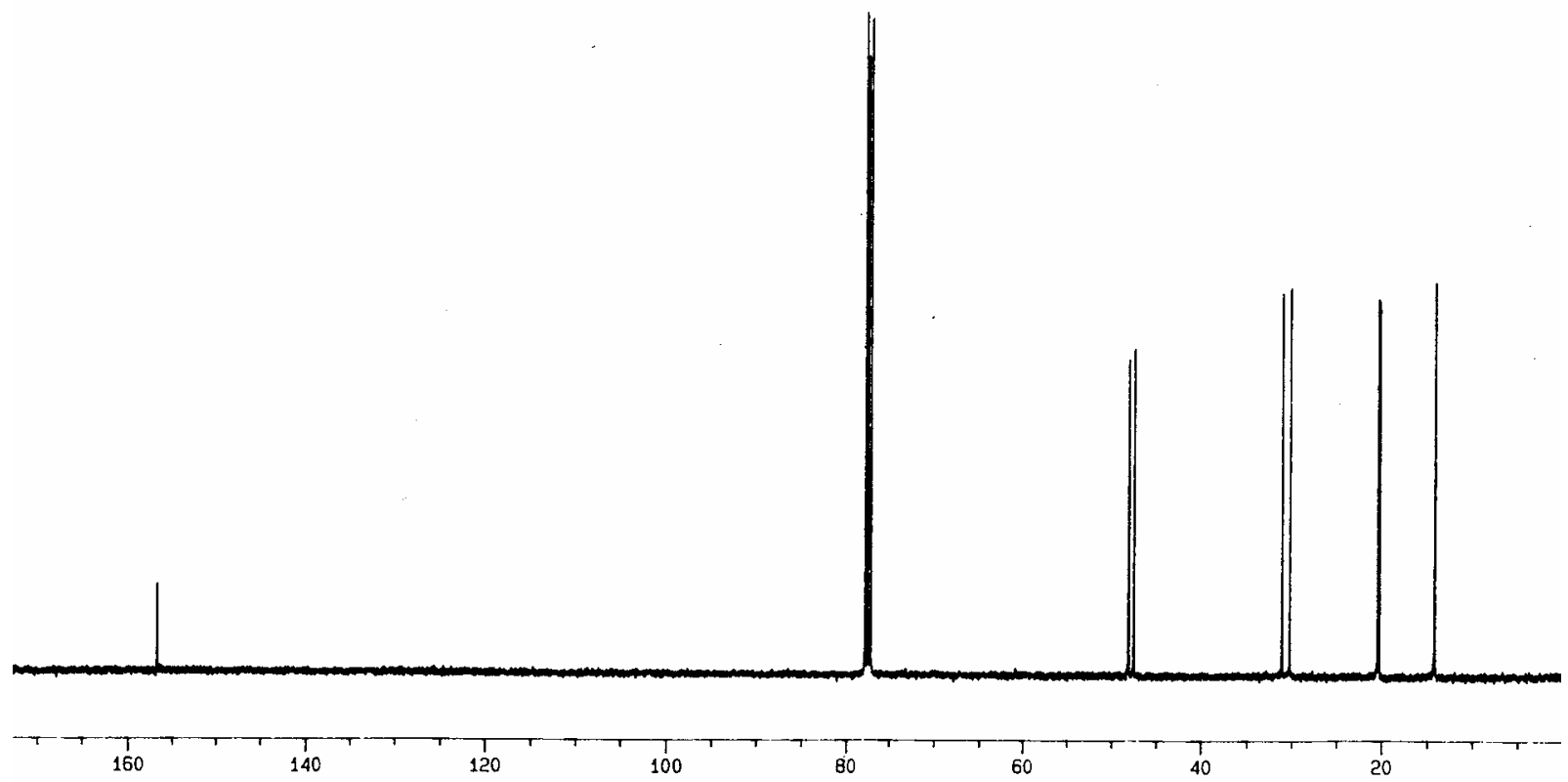




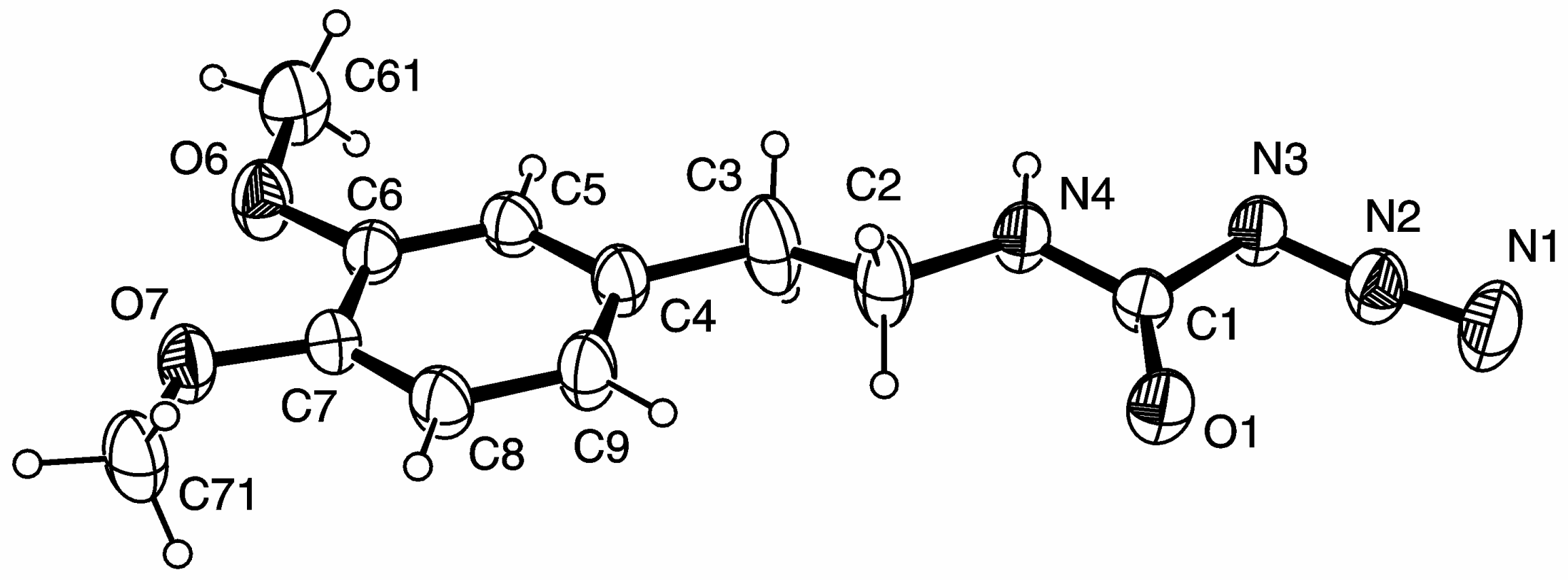

Ortep plot of $\mathbf{2 e}$ 


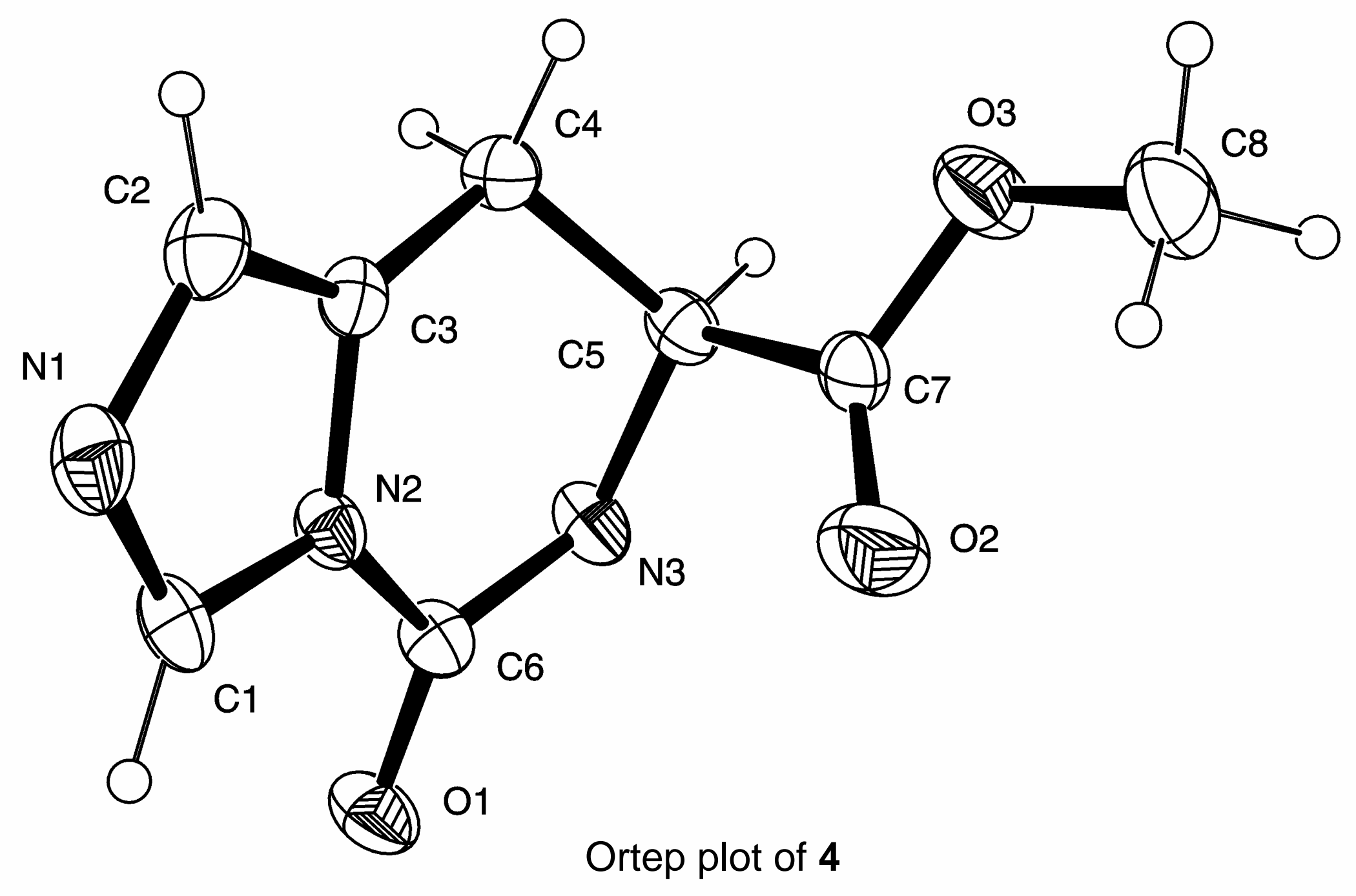

